\title{
Phylogenetic analysis of Fritillaria cirrhosa D. Don and its closely related species based on complete chloroplast genomes
}

\author{
Qi Chen ${ }^{1}$, Xiaobo Wu ${ }^{1}$, Dequan Zhang ${ }^{\text {Corresp. } 1,2}$ \\ ${ }^{1}$ College of Pharmacy and Chemistry, Dali University, Dali, Yunnan, China \\ 2 Institute of Materia Medica, Dali University, Dali, Yunnan, China \\ Corresponding Author: Dequan Zhang \\ Email address: zhangdeq2008@126.com
}

Fritillaria cirrhosa D. Don, whose bulb is used in a well-known traditional Chinese medicine to relieve cough and eliminate phlegm, is one of the most important medicinal plants of Fritillaria L. The species is widely distributed among the alpine regions in southwestern China and possesses complex morphological variations in different distributions. A series of newly related species were reported, based on obscure morphological differences. As a result, $F$. cirrhosa and its closely related species constitute a taxonomically complex group. However, it is difficult to accurately identify these species and reveal their phylogenetic relationships using traditional taxonomy. Molecular markers and gene fragments have been adopted but they are not able to afford sufficient phylogenetic resolution in the genus. Here, we report the complete chloroplast genome sequences of $F$. cirrhosa and its closely related species using next generation sequencing (NGS) technology. Eight plastid genomes ranged from $151,058 \mathrm{bp}$ to $152,064 \mathrm{bp}$ in length and consisted of 115 genes. Gene content, gene order, GC content, and IR/SC boundary structures were highly similar among these genomes. SSRs and five large repeat sequences were identified and the total number of them ranged from 73 to 79 and 63 to 75, respectively. Six highly divergent regions were successfully identified that could be used as potential genetic markers of Fritillaria. Phylogenetic analyses revealed that eight Fritillaria species were clustered into three clades with strong supports and F. cirrhosa was closely related to $F$. przewalskii and F. sinica. Overall, this study indicated that the complete chloroplast genome sequence was an efficient tool for identifying species in taxonomically complex groups and exploring their phylogenetic relationships. 
1 Phylogenetic analysis of Fritillaria cirrhosa D. Don and

2 its closely related species based on complete

3 chloroplast genomes

4

5 Qi Chen ${ }^{1}$, Xiaobo $\mathrm{Wu}^{1}$, Dequan Zhang ${ }^{1,2, *}$

$6{ }^{1}$ College of Pharmacy and Chemistry, Dali University, Dali, China.

$7 \quad{ }^{2}$ Institute of Materia Medica, Dali University, Dali, China

8 *Correspondence: Dequan Zhang zhangdeq2008@126.com

9

10

\section{Abstract}

Fritillaria cirrhosa D. Don, whose bulb is used in a well-known traditional Chinese medicine to relieve cough and eliminate phlegm, is one of the most important medicinal plants of Fritillaria L. The species is widely distributed among the alpine regions in southwestern China and possesses complex morphological variations in different distributions. A series of newly related species were reported, based on obscure morphological differences. As a result, F. cirrhosa and its closely related species constitute a taxonomically complex group. However, it is difficult to accurately identify these species and reveal their phylogenetic relationships using traditional taxonomy. Molecular markers and gene fragments have been adopted but they are not able to afford sufficient phylogenetic resolution in the genus. Here, we report the complete chloroplast genome sequences of $F$. cirrhosa and its closely related species using next generation sequencing (NGS) technology. Eight plastid genomes ranged from 151,058 bp to 152,064 bp in length and consisted of 115 genes. Gene content, gene order, GC content, and IR/SC boundary structures were highly similar among these genomes. SSRs and five large repeat sequences were identified and the total number of them ranged from 73 to 79 and 63 to 75 , respectively. Six highly divergent regions were successfully identified that could be used as potential genetic markers of Fritillaria. Phylogenetic analyses revealed that eight Fritillaria species were clustered into three 
27 clades with strong supports and F. cirrhosa was closely related to F. przewalskii and F. sinica.

28 Overall, this study indicated that the complete chloroplast genome sequence was an efficient tool

29 for identifying species in taxonomically complex groups and exploring their phylogenetic 30 relationships.

31 Subjects Genomics, Molecular Biology, Plant Science

32 Key words Fritillaria cirrhosa D. Don, complete chloroplast genome, closely related species,

33 taxonomically complex groups, phylogenetic relationship 


\section{Introduction}

Fritillaria L. is one of the most important genera in Liliaceae, which includes approximately 140 species of perennial herbaceous plants (Day et al., 2014; Tekşen et al., 2010). Almost all of the species are distributed in the temperate regions of the northern hemisphere (Rix et al., 2001). There are 24 species in China and most of them possess important medicinal properties, these species include F. cirrhosa D. Don., F. ussuriensis Maxim., F. walujewii Regel., F. thunbergii Miq., and others (Chen \& Helen, 2000; National Pharmacopoeia Committee, 2015). Of these species, $F$. cirrhosa is one of the major original plants of Fritillariae Cirrhosae bulbus, a famous traditional Chinese medicine, which is used to relieve cough and eliminate phlegm (National Pharmacopoeia Committee, 2015). It is mainly found in areas of high altitude in the southwest of China and grows in moist environments near bushes, meadows, and other similar habitats (Chen \& Helen, 2000). However, this species exhibits complicated variations in morphology in different regions, especially in flower color and apex shape of bracts. Based on uncertain morphological differences, a series of newly related species were reported. As a result, $F$. cirrhosa and its closely related species constitute a taxonomically complex group that is difficult to be clearly distinguished based on morphological traits alone (Luo \& Chen, 1996; Zhang et al., 2001; Zhang \& Cheng,1998). Luo \& Chen (1996) had proposed the concept of a "complex group of F. cirrhosa" which was composed of four species, namely F. cirrhosa, F. sichuanica, $F$. taipaiensis and F. yuzhongensis based on uncertain morphological characteristics and geographical distributions. They also pointed out that $F$. sichuanica was possibly a hybrid among F. cirrhosa, F. przewalskii and F. unibracteata. This posits the question of whether the theory is reasonable and what would then be the phylogenetic relationships of the species in the so-called "complex group of $F$. cirrhosa" and their close relatives. 
Over the past few decades, molecular methods have been widely used in plant evolution and phylogeny due to rapid development of molecular technologies. As a result, the well-known APG (Angiosperm Phylogeny Group) classification system was constructed based on the latest progress in plant molecular phylogenetics (Byng et al., 2016; The Angiosperm Phylogeny Group, 1998; 2003; 2009). Although molecular markers were also used for phylogenetic inference in complicated groups (Simmons et al., 2007), only a few species of Fritillaria adopted them to explore phylogeny (Çelebi et al., 2008; Wietsma et al., 2015). Nevertheless, gene fragments, especially nrITS and cpDNA genes, gained more attention due to the rapid development of DNA sequencing (Gao et al., 2010; Kress et al., 2005; Mishra et al., 2015). Day et al. (2014) elaborated on the evolutionary relationships of 92 Fritillaria species based on three plastid regions but most of the species were not well distinguished. Huang et al. (2018) used three plastid markers (matK, $r b c L$ and $r p l$ 16) and nuclear ITS to explore the phylogeny with 191 taxa in the tribe Lilieae (including 57 Fritillaria species) but the boundaries between a few species of Fritillaria remained ambiguous and needed further research. Meanwhile, the combination of nrITS and cpDNA genes was also adopted to reveal inter-specific relationships and to discriminate between the species of Fritillaria. Although these gene fragments had a preliminary resolution on certain species in Fritillaria, they could not be effective in discriminating between the closely related species (Khourang et al., 2014; Rønsted et al., 2005; Zhang et al., 2016). Overall, it is probably sufficient to use individual or combined regions based on Sanger sequencing in order to explore the phylogenetic relationships of major genera, but they are generally insufficient for complex groups or closely related species (Liu et al., 2017). Fortunately, with the emergence and development of next-generation sequencing (NGS), the complete chloroplast genome might be a better tool for discriminating between species and revealing the phylogenetic relationships of complex groups (Mardis, 2008; Parks et al., 2009; Shendure \& Ji, 2008; Tangphatsornruang et al., 2009).

In plants, chloroplasts (cp) are photosynthetic organelles providing the necessary energy for growth and are fundamental in the biosynthesis of starch, fatty acids, pigments, and amino acids 
108 (Gao et al., 2010; Neuhaus \& Emes, 2000). Typically, angiosperm chloroplasts have a circular genome ranging from 72 to $217 \mathrm{~kb}$ and quadripartite structure composed of a large single copy region (LSC), a small single copy region (SSC), and a pair of inverted repeats (IRs) (Moore et al., 2010; Sugiura, 1992; Wang et al., 2015; Yurina \& Odintsova, 1998). In contrast with nuclear and mitochondrial genomes, cp genomes are largely conserved in the gene content, organization, and structure. Moreover, they are typically inherited maternally in the angiosperm, which is beneficial in genetic engineering due to the lack of cross-recombination (Maliga, 2002; Tangphatsornruang et al., 2009). The initial cp genomes were sequenced from tobacco and liverwort (Ohyama et al., 1986; Shinozaki et al., 1986). The plastid genome exhibits an overwhelming superiority for use in species discrimination of complex taxa and has been widely used to reveal their unresolved phylogenetics (Bayly et al., 2013; Du et al., 2017; Henriquez et al., 2014), such as in Araceae, Arundinarieae, Lemnoideae, Myrtaceae, Nelumbonaceae, Amborella, Nymphaea, Citrus, Gossypium and Oncidium (Bayly et al., 2013; Carbonell-caballero et al., 2015; Ding et al, 2017; Goremykin et al., 2003, 2004; Henriquez et al., 2014; Li et al., 2014a; Ma et al., 2014; Wu et al., 2010; Xue et al., 2012). In a recent study, Park et al. (2017) evaluated the relationships between $F$. ussuriensis and $F$. cirrhosa based on the chloroplast genome. Bi et al. (2018) explored the phylogenetic relationship of eight species representing each subgenus of Fritillaria using the complete chloroplast genome. Li et al. (2018) also adopted the plastid genomes to reveal inter-specific relationships among seven Fritillaria species that were mainly distributed in the Xinjiang province in China. Although these studies had partly revealed the classification and phylogenetics of Fritillaria and showed the power of a complete chloroplast genome, F. cirrhosa and its closely related species were not included and their relationships were still unresolved. Hence, we attempted to report the complete chloroplast genomes of $F$. cirrhosa and its related species, as well as to explore their phylogenetic relationships.

Here, we presented complete chloroplast genome sequences of several Fritillaria species using Illumina sequencing technology and performed comparative analyses of genomic 
135 information. Our aims were as follows: (1) to investigate the global structure patterns of eight

136 plastid genomes in Fritillaria in this study; (2) to examine the variations of simple sequence

137 repeats (SSRs) and other repeats (tandem, palindrome, forward, reverse, and complement repeats)

138 among the eight Fritillaria plastid genomes; (3) to discover highly divergent regions that could

139 be used as specific DNA barcodes for Fritillaria; and (4) to reveal phylogenetic relationships 140 between $F$. cirrhosa and its closely related species. The study might provide better understanding 141 of phylogenetic relationships of the complex group and afford sufficient genomic information to 142 use in further research and the application of these medicinal species.

\section{Materials \& Methods}

\section{Material sampling}

Eight species, including Fritillaria cirrhosa and its closed relatives were collected and used in this study (Fig. 1, Table S1). F. thunbergii was used as a supplemental outgroup for phylogenetic analysis. The related species were collected in the wilds of the Hengduan Mountains but $F$. thunbergii was cultivated in the Zhejiang province in China. Fresh, unblemished leaves were sampled from healthy, mature individuals and then dried with allochroic silicagel during the field work. Meanwhile, 3-5 individuals with flowers were collected and preserved as voucher specimens that were then used for morphological analysis and taxonomic identification. During the field work, geographic information, such as latitude, longitude, and altitude etc. was determined by Global Position System (GPS, Garmin) and morphologic traits (especially of the flower) were described immediately. All voucher specimens of Fritillaria were identified carefully by Dr. Dequan Zhang and deposited at the Herbarium of Medicinal Plants and Crude Drugs of the College of Pharmacy and Chemistry, Dali University.

\section{DNA extraction, sequencing, and assembly}

Total genomic DNA was extracted from about $100 \mathrm{mg}$ of dried leaf material according to a modified CTAB method (Doyle, 1987; Yang et al., 2014). DNA quality was checked by 
162 electrophoresis on $1.2 \%$ agarose gel and then its concentration was determined using 163 SmartSpecTM Plus Spectrophotometer (Bio-Rad, Hercules, CA, USA). DNA extracts were 164 fragmented for $300 \mathrm{bp}$ short-insert library construction and sequenced $-2 \times 150 \mathrm{bp}$ paired-end 165 (PE) reads on an Illumina HiSeq X-Ten instrument at Beijing Genomics Institute (BGI, 166 Shenzhen, China).

167 The raw data was filtered using Trimmomatic v.0.32 (Bolger et al., 2014) with default 168 settings. Then paired-end reads of the clean data were filtered and assembled into contigs using 169 GetOrganelle.py (Jin et al., 2018) with Fritillaria cirrhosa (accession number: KF769143) as a

170

171

172

reference (Li et al., 2014b), calling the bowtie2 v., blastN v. and SPAdes v.3.10 (Bankevich et al., 2012). The de novo assembly graphs were visualized and edited using Bandage Window dynamic v.8.0 (Wick et al., 2015) and then a whole or nearly whole circular chloroplast genome was generated.

\section{Genome annotation and sequence submission}

The plastid genomes were annotated by aligning to the complete chloroplast genome sequence published in GenBank (Fritillaria cirrhosa, accession number: KF769143) using MAFFT (Katoh \& Standley, 2013) with default parameters, coupled with manual adjustment using Geneious v.10.1.3 (Kearse et al., 2012). The circular genome map was generated with OGDRAW v.1.2 (Lohse et al., 2013). Finally, the annotated chloroplast genomes of the nine Fritillaria species were submitted to GenBank (Table 1, Table S1).

\section{Genome Comparative Analysis}

In this study, the multiple sequence alignment of chloroplast genome sequences was performed using MAFFT v.7.129 with default settings and adjusted manually in BioEdit v.7.0.9 (Hall, 1999; Katoh \& Standley, 2013). The mVISTA software was used to compare the complete chloroplast genome of $F$. cirrhosa with eight other Fritillaria species, taking annotation of the chloroplast genome of $F$. cirrhosa (accession number: KF769143) as a reference. Default 
parameters were utilized to align the chloroplast genomes in Shuffle-LAGAN mode (Frazer et al., 2004). P-distance, GC content, and codon use were analyzed by the software MEGA v.7.0.26 (Kumar et al., 2016). DnaSP v.6.11 (Rozas et al., 2017) was adopted to calculate the variable and parsimony information sites and nucleotide diversity of five regions (whole chloroplast genome, large single copy, small single copy, inverted repeat regions, and protein coding genes). Additionally, the IR contraction/expansion regions were compared among the eight Fritillaria species.

\section{Characterization of repeat sequences and SSRs}

REPuter software was used to identify repeat sequences, including palindromic, complement, reverse, and forward repeats within the chloroplast genome. The following conditions for repeat identification were used in the analysis: (i) Hamming distance of 3, (ii) $90 \%$ or greater sequence identity, and (iii) a minimum repeat size of 30bp (Kurtz et al., 2001). MISA was adopted to evaluate SSRs. The minimum thresholds were set to ten repeat units for mononucleotide SSRs, five repeat units for dinucleotide SSRs, four repeat units for trinucleotide, and three repeat units for tetranucleotide, pentanucleotide, hexanucleotide SSRs (Murat et al., 2011). In addition, tandem repeats in eight Fritillaria species chloroplast genomes were identified using Tandem Repeats Finder v.4.09 with the following settings: 80, 10, 50, and 500 for match probability, indel probability, minimum alignment score, and maximum period size, respectively (Benson, 1999).

\section{Chloroplast genome analysis by sliding window}

After using MAFFT v.7.129 to align the chloroplast genome sequences, BioEdit software was used to adjust the sequences manually (Hall, 1999; Katoh \& Standley, 2013). A sliding window analysis was conducted for nucleotide variability (Pi) in the whole chloroplast genome using the DanSP. The step size was set to 200 bp, with a 600 bp window length (Rozas et al., 2017). 


\section{Phylogenetic analyses}

218 The eight species of $F$. cirrhosa and its closely related species were used for phylogenetic analysis, to be supplemented with $F$. thunbergii (accession number: MH244914) and Lilium brownii F. E. Brown ex Miellez (accession number: NC_035588) as outgroups (Du et al., 2017). Furthermore, the available chloroplast genome sequence of F. unibracteata var. wabuensis (KF769142), which was a variety of $F$. unibracteata, was downloaded from GenBank for our phylogenetic analysis (Li et al., 2016). Phylogenetic inference was performed based on the following five data sets: (1) chloroplast genome sequence (only containing one IR), (2) large single copy region, (3) small single copy region, (4) inverted repeat region, and (5) proteincoding genes. The sequences were aligned using MAFFT and then edited by BioEdit manually (Hall, 1999; Katoh \& Standley, 2013). Lengths of aligned sequences were shown in Table S8. In order to explore the phylogenetic relationship of $F$. cirrhosa and its closely related species, Bayesian inference (BI), Maximum parsimony (MP) and Maximum likelihood (ML) methods were adopted for phylogenetic inference, respectively.

MEGA v.7.0.26 was used for MP analysis with 1,000 bootstrap replicates (Kumar et al. 2016). For BI and ML analysis, the best substitution models were tested based on Akaike information criterion (AIC) by jModelTest v.2.1.7 (Darriba et al., 2012). Best-fitting models in this analysis were GTR $+\mathrm{I}+\mathrm{G}$ for LSC and SSC region, and GTR $+\mathrm{I}$ for others (Table S8). ML analysis was performed with RAxML v.8.2.4 (Stamatakis, 2014). And 1,000 replications were adopted to calculate the local bootstrap probability of each branch. BI analysis was conducted in MrBayes v.3.2.6 (Ronquist et al., 2012). The Markov Chain Monte Carlo (MCMC) algorithm was calculated for $1,000,000$ generations with a sampling of trees every 1,000 generations. The first $25 \%$ of generations were discarded as burn-in. Stasis was considered to be reached when the average standard deviation of split frequencies was $<0.01$ and a consensus tree was constructed using the remaining trees. 
243

244

245

246

247

248

249

250

251

252

253

254

255

256

257

258

259

260

261

262

263

264

265

266

267

268

\section{Results}

\section{Chloroplast genome organization of Fritillaria chloroplast genomes}

Nucleotide sequences of the eight Fritillaria chloroplast genomes ranged from 151,083 bp in F. unibracteata to $152,064 \mathrm{bp}$ in $F$. sinica and shared the typical quadripartite structure, composed of a pair of IRs (26,090-26,364 bp) separated by the LSC (81,339-81,827 bp) and SSC (17,526-17,545 bp) regions (Table 1, Fig. 2). GC content of the complete chloroplast genomes was $36.9 \%-37.0 \%$ (Table 1). The content of the IR regions $(42.5 \%)$ was higher than that of whole genome (36.9\%), LSC (34.9\%), and SSC (30.5\%) in F. cirrhosa due to the presence of eight rRAN (55\%) sequences in these regions (Table 2).

In the eight whole chloroplast genomes, a total of 115 genes were found, including 78 protein coding genes, 30 tRNA genes, 4 rRNA genes, and 3 pseudogenes (infA, $y c f 15$ and $y c f 68$ ) (Table 1, Fig. 2). The protein coding genes present in the chloroplast genome of eight Fritillaria genomes included nine genes for large ribosomal proteins ( $r p l 2$, rpl14, rpl16, rpl20, rpl22, rpl23, rpl32, rpl33, rpl36), 12 genes for small ribosomal proteins (rps2, rps3, rps4, rps $7, r p s 8, r p s 11$, rps12, rps14, rps15, rps16, rps18, rps19), 5 genes for photosystem I (psaA, psaB, psaC, psaI, $p s a J), 15$ genes for photosystem II ( $p s b A, p s b B, p s b C, p s b D, p s b E, p s b F, p s b H, p s b I, p s b J, p s b K$, $p s b L, p s b M, p s b N, p s b T, p s b Z)$, and 6 genes for ATP synthase (atpA, atpB, atpE, atpF, atpH, atpI) (Table 3, Fig. 2). Furthermore, 20 duplicated genes were found in the IR regions, as well as 5 protein coding genes, 11 tRNA genes and 4 rRNA genes. 26 protein coding genes possessed introns (Fig. 2).

Protein coding genes, rRNA and tRNA were encoded by $44.89 \%, 5.96 \%$, and $1.89 \%$ in the $F$. cirrhosa whole chloroplast genome, respectively, and the remaining $47.26 \%$ was non-coding regions. The 20 amino acids crucial for protein biosynthesis were encoded by 30 tRNA. Moreover, protein coding genes included 78 protein genes and the length was $68,234 \mathrm{bp}$, which comprised 22,396 codons (Table 2). Interestingly, among all of the encoded amino acids, leucine $(10.32 \%)$ and cysteine $(1.57 \%)$ were the maximum and minimum commonly detected amino 
269

270

271

\section{2}

273

274

275

276

277

278

279

280

281

282

283

284

285

286

287

288

289

290

\section{1}

292

293

294

295

acids, respectively (Table S2). Within the protein coding regions, the AT percentages for the first, second, and third codons were 55.5\%,62.0\% and 70.4\% in F. cirrhosa, respectively (Table 2).

\section{SSR analysis of Fritillaria chloroplast genomes}

Numerous SSR loci were found through the MISA analysis of nine Fritillaria chloroplast genome sequences. In total, five types of SSR (mononucleotide, dinucleotide, trinucleotide, tetranucleotide, and pentanucleotide repeats) were detected based on the comparison of eight Fritillaria cp genomes. A total of 78 perfect SSRs were found in F. cirrhosa (Fig. 3A). Similarly, 73, 74, 78, 79, 77, 76, and 75 SSRs were detected in F. sichuanica, F. przewalskii, $F$. unibracteata, F. taipaiensis, F. yuzhongensis, F. sinica, and F. dajinensis. Lengths of those SSRs ranged from 10 to $22 \mathrm{bp}$ (Table S3). The most abundant type of SSR were mononucleotide repeats ranging from $51 \mathrm{bp}$ in $F$. sichuanica to $56 \mathrm{bp}$ in $F$. unibracteata, followed by dinucleotide repeats, tetranucleotide repeats, trinucleotide repeats, and pentanucleotide repeats (Fig. 3A). In the cp genome of F. cirrhosa, all mononucleotide repeats are composed of A $(47.27 \%)$ and T (52.72\%) motifs in the majority of dinucleotide SSRs are AT (64.29\%) (Fig. 3B).

Further analysis revealed that most of the microsatellites were located in the LSC region, with a small portion distributed through the SSC and IR regions (Fig. 3C). Moreover, the SSRs in the genomes were distributed mainly in the intergenic spacer (IGS), with others dispersed at similar levels in introns and protein coding genes (CDS) (Fig. 3D). Seven protein coding genes in the SSR loci were $r p o C 2, \operatorname{cem} A, n d h D, n d h G, n d h H, y c f 1$, and $y c f 2$ in the CDS regions of the Fritillaria cp genome (Table S3).

\section{Other repeats analysis of Fritillaria chloroplast genomes}

A total of 63 repeats including tandem, palindrome, forward, reverse, and complement repeats were found in the $F$. cirrhosa chloroplast genome. Similarly, 65, 66, 70, 66, 73, 75, and 73 repeats were detected in F. sichuanica, F. przewalskii, F. unibracteata, F. taipaiensis, $F$. yuzhongensis, F. sinica, and F. dajinensis, respectively (Fig. 4A). Among these, tandem repeats, 
296

297

298

299

300

301

302

303

304

305

306

307

308

309

310

311

312

313

314

315

316

317

318

319

320

321

322

which had larger numbers than others, were mainly distributed in the intergenic spacer (IGS), with others dispersed in protein coding genes (CDS) and introns (Fig.4B). The tandem repeats in the CDS regions were located in five protein coding genes $(\operatorname{trnK}-U U U, \operatorname{rps} 11, \operatorname{rps} 16, y c f 1$, and $y c f 2$ ) of the plastid genomes (Table S4) and mainly ranged from 10 to $29 \mathrm{bp}$ in length, whereas only one tandem repeat longer than $40 \mathrm{bp}$ was found in the F. sichuanica genome (Fig. 4C). In the remaining four repeats, most occurred in the regions of the intergenic spacer, whereas some were found in the protein coding genes and intron (Table S5). Copy lengths with 30-44 bp were the most common. Moreover, the length of palindrome repeats more than $90 \mathrm{bp}$ were found in four plastid genomes (F. sichuanica, F. przewalskii, F. yuzhongensis, and F. sinica). However, almost all of the lengths of the forward and reverse repeats were less than 59 bp in eight Fritillaria chloroplast genomes (Fig. 4D-4F, Tables S4-S6).

\section{Comparison of chloroplast genome among $F$. cirrhosa and related species}

The annotation of $F$. cirrhosa (accession number: KF769143) was used as a reference for visualization analysis of the pairwise chloroplast genomic alignment between $F$. cirrhosa and its closely related species using mVISTA (Fig. 5). The alignment revealed a high sequence similarity across eight Fritillaria plastid genomes, which showed that the genomes were highly conserved. Furthermore, a vast majority of sequence variations were concentrated in the single copy regions, compared with the least number in the IR regions. This indicated that there were higher divergence levels in the single copy regions than that in the IR regions. Moreover, coding regions were less divergent than non-coding regions. Similarly, sequence divergence in the intron was higher than that in the exon. Highly divergent regions among eight Fritillaria chloroplast genomes were mainly located in the intergenic spacers, including atpH-atpI, rpoBtrnC-GCA, petN-psbM, psbM-trnD-GUC, trnT-GGU-psbD, trnS-GGA-rps4, trnT-UGU-trnL$U A A$, accD-psaI, $y c f 4$-cemA, and psbE-petL, but others (matK and $y c f 1$ ) were distributed in protein coding regions.

Expansion and contraction at the boundaries of IR regions of eight Fritillaria chloroplast 
323

324

325

326

327

328

329

330

331

332

333

334

335

336

337

338

339

340

341

342

343

344

345

346

347

348

349

genomes were revealed and a detailed comparison of four junctions of two IRs between $F$. cirrhosa and its closely related species was performed (Fig. 6). There were some differences in length compared with each region among the Fritillaria chloroplast genomes, but they exhibited striking similarities on the IR borders. Although IR regions were highly conserved, subtle structure variation was still observed in the chloroplast genomes. In contrast, $y c f 1$ was mainly located in the SSC region ranging from $4293 \mathrm{bp}$ to $4320 \mathrm{bp}$ and others $1230 \mathrm{bp}$ in IRa region. The border between IRb/LSC extended into the rps19, but there were only $31 \mathrm{bp}$ in the IRb region of F. cirrhosa. Moreover, variation was found in F. unibracteata, and $n d h F$ was 24 bp away from the SSC/IRb border.

Evolutionary divergences and differences among the eight Fritillaria chloroplast genomes were compared using sequence distance and nucleotide substitutions. Across all the species, pdistance was 0.0003-0.0023, and the value of the nucleotide differences was 52-340 (Table 4). The p-distance in three Fritillaria (F. cirrhosa, F. przewalskii, and F. sinica) was between 0.0005-0.0008.

\section{Divergence region in chloroplast genome of the $F$. cirrhosa and related species}

Nucleotide diversity of highly variable regions was calculated with a sliding window (step size was set to $200 \mathrm{bp}$, with a $600 \mathrm{bp}$ window length) to estimate the divergence level of different regions in the eight Fritillaria plastid genomes. Of these, the SSC region exhibited the highest divergence levels (0.00332) and IR regions had the least (0.00038) (Table 5). Furthermore, six regions with a relatively high variability, including 5 intergenic regions (trnS-GCU-trnR-UCU, rpoB-psbD, rps4-trnF-GAA, petA-psbL, and $n d h F-n d h D)$ and one gene region (ycfl) from the genomes, were selected as potentially suitable gene fragments for the study of species identification and phylogenetics in Fritillaria (Fig. 7). All highly divergent sequences were found in the SC regions whereas no higher variable loci were found in the IR regions. The six highly variable regions included 257 variable sites which possessed 116 parsimony informative sites and their nucleotide diversity values ranged from 0.00455 to 0.00935 (Table S7). The petA- 
350

351

352

353

354

355

356

357

358

359

360

361

362

363

364

365

366

367

368

369

370

371

372

373

374

375

376

$p s b L$ showed the highest variability, the next more variable regions were $\operatorname{rps} 4-\operatorname{trn} F-G A A, n d h F$ $n d h D, y c f 1$ and $r p o B-p s b D$, but that of $\operatorname{trnS}-G C U-\operatorname{trn} R-U C U$ was the lowest.

\section{Phylogenetic relationship of $F$. cirrhosa and related species}

In this study, five datasets extracted from the eleven plastid genomes were used for phylogenetic analysis (Fritillaria thunbergii and Lilium brownie were used as outgroups). BI, MP, ML analyses were performed to construct phylogenetic trees using the datasets (Table S8) and topology structures of the previous three trees were nearly identical. Finally, the BI tree was adopted to present phylogenetic results, with the addition of support values from MP and ML analyses. The phylogenetic tree based on different datasets achieved higher support values, except the IR dataset (Fig. 8, Fig. S1). According to the trees, the eight species of Fritillaria were obviously divided into three clades (clade I, II and III). Clade I contained four species with strong support, namely $F$. sichuanica, $F$. dajinensis, $F$. unibracteata, and $F$. unibracteata var. wubuensis. It was revealed that $F$. sichuanica had a close relationship with $F$. dajinensis. Fritillaria taipaiensis, and F. yuzhongensis, both of which were distributed in the northern edge of the complex group and were clustered into one clade (II). The last clade (III) was composed of $F$. cirrhosa, F. przewalskii and $F$. sinica which revealed that $F$. cirrhosa was a sister species to the latter two species.

\section{Discussion}

\section{Comparative analysis of Fritillaria chloroplast genomes}

Eight plastid genomes in this study ranged from 151,009 bp to 152,064 bp, consisting of 115 genes with a GC content of 36.9\%-37.0\% (Table 1, Table 2 and Fig. 2). In the chloroplast genome of $F$. cirrhosa, the GC content of the IR regions (42.5\%) was highest, which could be attributed to the presence of eight rRNA (55\%) sequences in these regions (Table 2). The present results were similar to previous reports with a higher GC content in the IR regions (Bi et al., 2018; Li et al., 2018; Park et al., 2017). These studies might be beneficial for systematically 
377

378

379

380

381

382

383

384

385

386

387

388

389

390

391

392

393

394

395

396

397

398

399

400

401

402

403

recognizing the gene number, gene order, and chloroplast genome structure of Fritillaria. Furthermore, protein coding genes of the F. cirrhosa genome were encoded by $44.89 \%$ and the AT percentage of the third codon in them was $70.4 \%$. Preference for a higher AT content at the third codon position has been also observed in other terrestrial plant chloroplast genomes (Asaf et al., 2016; Liu \& Xue, 2005; Qian et al., 2013; Tangphatsornruang et al., 2009).

SSRs in the chloroplast genome (cpSSRs), which are 1-6 bp repeating sequences and distributed throughout the genome, have been used for the study of population genetics because of their high variability (Asaf et al., 2016; Pauwels et al., 2012; Powell et al., 1995). In this study, certain parameters were set as microsatellites of more than $10 \mathrm{bp}$ are prone to slipped-strand mispairing (Raubeson et al. 2007; Rose \& Falush, 1998). Five types of SSR (mononucleotide, dinucleotide, trinucleotide, tetranucleotide, and pentanucleotide repeats) were detected and number of them ranged from 73 to 79 (Fig. 3). The detected SSRs were located in seven protein coding genes (rpoC2, cemA, $n d h D, n d h G, n d h H, y c f 1$ and $y c f 2)$ of the Fritillaria plastid genomes. In the previous study, Bi et al. (2018) observed that five types of SSRs were located in nine protein coding genes (matK, rpoC1, rpoC2, cemA, $n d h D, n d h G, n d h H, y c f 1$ and $y c f 2)$. Lu et al. (2016) found that 15 SSRs were located in eight protein coding genes (rpoC2, cemA, rpl22, $n d h D, n d h E, n d h H, y c f 1$ and $y c f 2$ ) of three cardiocrinum plastid genomes. Therefore, all the studies strongly indicated that the chloroplast genome could be used for developing lineagespecific cpSSR markers that could help for studies on population genetics of the Fritillaria species.

Repeat sequences play an important role in genome rearrangement and variation due to the illegitimate recombination and slipped-strand mispairing in the chloroplast genome (CavalierSmith, 2002; Lu et al., 2016; Yuan et al., 2017). In the present repeat analysis, five types of repeats including tandem, palindrome, forward, reverse, and complement repeats were identified (Fig. 4). Among them, tandem repeats had the largest numbers and were mainly distributed in the intergenic spacer (IGS). Although substantial repeats have been distinguished in the chloroplast genome of higher plants, the mechanism for the origin of these tandem repeats was unclear 
404 (Vieira et al., 2014; Yi et al., 2013). Significant correlations have been observed among DNA

405

406

407

408

409

410

411

412

413

414

415

416

417

418

419

420

421

422

423

424

425

426

427

428

429

430

rearrangement, mutation, and gene duplication (Cosner et al., 1997; Do et al., 2014; Vieira et al., 2014; Yi et al., 2013). It was reported that repeat sequences made sense for population genetics because of their significance in rearrangement (Cavalier-Smith, 2002). Most of the remaining four repeats occurred in intergenic spacer regions and the lengths ranged from 9 to 95 (Fig. 4D4F, Tables S5-S6). The results for the locations and sequence lengths of the four major repeats were similar to the latest studies (Bi et al., 2018; Park et al., 2017). The research also revealed that repeat sequences were caused by illegitimate recombination and slipped-strand mispairing in the genome (Cavalier-Smith, 2002; Lu et al., 2016; Yuan et al., 2017). Furthermore, the region where the repeats existed was a potential hotspot for genomic reconfiguration (Gao et al., 2009). Additionally, these repeat motifs might provide some informative sources to develop genetic markers for analysis on population genetics (Nie et al., 2012).

Expansion and contraction at the boundaries on the IR regions of the chloroplast genome are important factors that cause size variations and this plays a major role in structural stability and evolution (Asaf et al., 2018; Dang et al., 2014; Wang et al., 2008). In this study, a detailed comparison of four junctions of two IRs between $F$. cirrhosa and its closely related species was performed. The IR regions are highly conserved and structure variation was not significant in the eight Fritillaria chloroplast genomes (Fig. 6).

\section{Identification of highly variable regions}

Highly variable regions of the chloroplast genomes could not only be used for resolving phylogeny and identifying species at the species level, but also provide crucial information to explore species divergence and population structure at the population level (Dang et al., 2014; Du et al., 2017). Nucleotide diversity was calculated with a sliding window to estimate the divergence of different regions in eight Fritillaria cp genomes. Of these regions, the SSC region exhibited the highest value (0.00332) and the IR regions had the least (0.00038) (Table 5). Once again it indicated that IR regions were conserved in eight Fritillaria cp genomes. Similar results 
431

432

433

434

435

436

437

438

439

440

441

442

443

444

445

446

447

448

449

450

451

452

453

454

455

456

457

related to these regions have been reported in the latest studies of Fritillaria (Bi et al., 2018; Park et al., 2017) and have also been found in Lilium (Du et al., 2017). Furthermore, six relatively highly variable regions, including 5 intergenic regions $(t r n S-G C U-t r n R-U C U$, rpoB-psbD, rps4-

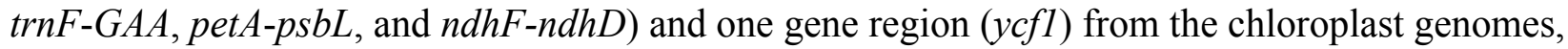
were selected as potentially suitable gene fragments to study species identification and phylogenetics in Fritillaria (Fig. 8). The region of petA-psbL possessed the highest variability, followed by rps4-trnF-GAA, ndhF-ndhD, ycfl and rpoB-psbD, whereas trnS-GCU-trnR-UCU was the lowest. Therefore, the regions with rich variation and suitable length, such as petA-psbL, rps4-trnF-GAA, ndhF-ndhD, ycfl and rpoB-psbD could be used as a prior choice of species identification for Fritillaria. Meanwhile, all of the highly variable regions are judged to be suitable for revealing phylogenetic relationships and genetic structure at the species and population level in Fritillaria.

\section{Phylogenetic analysis}

In the present study, the four datasets from the plastid genomes, unanimously clustered Fritillaria cirrhosa and its closely related species into three clades (clade I, II and III) based on BI, MP, and ML analysis (Fig. 7). First, clade I was composed of two parts: F. sichuanica and $F$. dajinensis, as well as $F$. unibracteata and its variety. It was surprising to find that $F$. sichuanica was so closely related to $F$. dajinensis because they were obviously different in flower traits (Fig. 1) (Chen \& Helen, 2000). One possible reason might be the conflict between molecules and morphology that was also observed in other taxa (Anand et al., 2016). The two species that were located at the northeastern edge of geographical distribution of the whole group, namely $F$. taipaiensis and F. yuzhongensis were clustered into clade II. Finally, F. cirrhosa was the most closely related to $F$. unibracteata and $F$. sinica. Although $F$. sichunica is thought to be a hybrid among $F$. cirrhosa, F. unibracteata and F. przewalskii (Luo \& Chen, 1996), they seemly did not show close relationships. Our results were highly supportive of those from other studies and agreed with Huang et al. (2018) in the phylogeny of Fritillaria at the species level. However, 
458

459

460

461

462

463

464

465

466

467

468

469

470

471

472

473

474

475

476

477

478

479

480

481

482

483

484

among $F$. sichunica and its relatives, the phylogenetic inference of these results remained ambiguous. Molecular data from the nuclear genome and genetic analysis on population level might be necessary to further explore phylogenetic relationships among these related species.

Moreover, this study preliminarily indicated that the concept of the "complex group of $F$. chirrhosa" suggested by Luo \& Chen (1996) might not contain four species but include other species as well (Fig. 8). Fritillaria cirrhosa is widely distributed in the alpine and subalpine regions of SW China, and exhibits complicated variations in morphology among the different distributions. Luo \& Chen (1996) proposed the concept of a "complex group of Fritillaria cirrhosa", including four species, namely F. cirrhosa, F. sichuanica, F. taipaiensis, and F. yuzhongensis based on obscure morphological traits and rough geographical distributions. However, there are no obvious borderlines among species within the complex group, as well as between the group and their closely related species. For example, F. sichunica is extremely similar to F. unibracteata except for subtle differences in length of the stigma lobes (Chen \& Helen, 2000). So, the concept might be unreasonable and should be revised based on more detailed research.

\section{Super and specific DNA barcodes}

Potential DNA barcodes are generally used in species identification and phylogenetic studies of plants, but they could not provide enough informative sites to resolve the relationships among F. cirrhosa and its closely related species (Burke et al., 2016; Percy et al., 2014; Zhang et al., 2016). In recent research, the complete chloroplast genome as a super-barcode has been proven to be an effective tool for species discrimination in some complicated groups, and specific DNA barcodes are a trade-off for species identification of those groups based on highly variable regions of the plastid genome (Chen et al., 2018; Ma et al., 2018). In the genus Fritillaria, the complete chloroplast genomes were much better at uncovering the phylogeny of Fritillaria species (Bi et al., 2018; Li et al., 2018; Park et al., 2017). Similarly, clear phylogenetic relationships among $F$. cirrhosa and its close relatives were indicated based on these tools with 
485

486

487

extremely high bootstrap values in this study (Fig. 8). Thus, using the whole chloroplast genome as a super-barcode might be suitable for the species identification of Fritillaria. Meanwhile, highly variable regions observed in this study could be also used as specific barcodes for identifying species in Fritillaria.

\section{Conclusion}

The chloroplast genomes of $F$. cirrhosa and its closely related species were sequenced using NGS technology and their genetic information was primarily revealed. The eight genomes exhibited a typical circular quadripartite structure and shared a high similarity in gene order and genomic structure, but still provided rich genetic information for research on the Fritillaria species. The position change of the IR/SC junction was not obvious among the eight cp genomes. SSRs, large repeat sequences, and pairwise sequence divergences were determined. Highly variable loci and divergent regions were identified as possible ways to develop genetic markers which could be used for further study on population genetics. Moreover, phylogenetic analyses revealed that the eight Fritillaria species were divided into three clades with high support values based on the genome-scale datasets. The results indicated that $F$. cirrhosa was the close relative to $F$. unibracteata and $F$. sinica; thus, it indicated that the concept of a "complex group of $F$. chirrhosa" might be inappropriate and need further revision. Furthermore, the complete chloroplast genomes and highly variable regions were very promising for identifying the species and resolving phylogeny in F. cirrhosa which meant that they could be used as super-barcode and specific barcodes of the genus. Overall, the study would be beneficial to facilitate our understanding on phylogeny and evolution in Fritillaria.

\section{Acknowledgements}

We thank Junbo Yang, Tingshuang Yi, Rong Zhang and Zhirong Zhang in Kunming Institute of Botany (Chinese Academy of Sciences, CAS) for their help in molecular experiment and data 
511 analysis of complete chloroplast genome in this study.

512

\section{Additional information and declarations}

514 Funding: This study was co-supported by the National Natural Science Foundation of China

515 (31660081), Yunnan Provincial Science and Technology Department (Grant No. 2016FB144).

\section{Competing Interests}

517 The authors declare there are no competing interests.

\section{Author Contributions}

519 Dequan Zhang conceived and designed the study.

520 Qi Chen analyzed the data and wrote the manuscript.

521 Xiaobo $\mathrm{Wu}$ helped to collect samples and perform experiments.

522 Dequan Zhang revised the manuscript finally.

\section{DNA Deposition}

524 The following information was supplied regarding the deposition of DNA sequences: The 9 525 complete chloroplast genomes in this paper were submitted to GenBank. Accession numbers are 526 MH244906-MH244914. The datasets used during the current study are available from the 527 corresponding author on reasonable request.

\section{Supplemental Information}

529 Fig. S1 Phylogenetic relationship of IR region of nine Fritillaria species. (A) Bayesian analysis 530 (BI), (B) maximum parsimony (MP), and (C) maximum likelihood (ML).

531 Table S1 Some information of ten Fritillaria species.

532 Table S2 Amino acid frequencies in protein coding genes of nine Fritillaria cp genomes.

533 Table S3 Distribution of simple sequence repeats (SSRs) loci in the nine Fritillaria chloroplast 534 genomes.

535 Table S4 Regions of tandem repeat in nine Fritillaria chloroplast genomes.

536 Table S5 A list of repeated sequences and their locations identified in the nine Fritillaria 
537

538

539

540

541

542

543

544

545

\section{6}

547

548

549

550

551

552

553

554

555

556

557

558

559

560

561

562

563

564

565

566

567

568

569

570

chloroplast genomes.

Table S6 Frequency of complement repeats by length in nine Fritillaria.

Table S7 Regions of highly variable sequences of Fritillaria.

Table S8 Length of each dataset matrix used for phylogeny construction and the best fitting models tested by jModeltest v.2.1.7 based on Akaike Information Criterion.

2

3

4

5

\section{References}

Anand KK, Jena SN, Chaudhary LB, and Singh M. 2016. Conflict between morphological and molecular data: A case study of Ficus krishnae (Moraceae). Phytotaxa 247:143-147. DOI 10.11646/phytotaxa.247.2.7

Asaf S, Khan AL, Khan AR, Muhammad W, Kang SM, Khan MA, Seok-Min L, and In-Jung L. 2016. Complete chloroplast genome of Nicotiana otophora and its comparison with related species. Frontiers in Plant Science 7. DOI 10.3389/fpls.2016.00843

Asaf S, Khan AL, Khan MA, Shahzad R, Lubna, Kang SM, Alharrasi A, Alrawahi A, and Lee IJ. 2018. Complete chloroplast genome sequence and comparative analysis of loblolly pine (Pinus taeda L.) with related species. Plos One 13:e0192966. DOI 10.1371/journal.pone.0192966

Bankevich A, Nurk S, Antipov D, Gurevich AA, Dvorkin M, Kulikov AS, Lesin VM, Nikolenko SI, Pham S, Prjibelski AD, Pyshkin AV, Sirotkin AV, Vyahhi N, Tesler G, Alekseyev MA, and Pevzner PA. 2012. SPAdes: a new genome assembly algorithm and its applications to single-cell sequencing. Journal of Computational Biology 19:455-477. DOI 10.1089/cmb.2012.0021

Bayly MJ, Rigault P, Spokevicius A, Ladiges PY, Ades PK, Anderson C, Bossinger G, Merchant A, Udovicic F, and Woodrow IE. 2013. Chloroplast genome analysis of Australian eucalypts - Eucalyptus, Corymbia, Angophora, Allosyncarpia and Stockwellia (Myrtaceae). Molecular Phylogenetics and Evolution 69:704-716. DOI 10.1016/j.ympev.2013.07.006

Benson G. 1999. Tandem repeats finder: a program to analyze DNA sequences. Nucleic Acids Research 27:573-580. DOI 10.1093/nar/27.2.573

Bi Y, Zhang MF, Xue J, Dong R, Du YP, and Zhang XH. 2018. Chloroplast genomic resources for phylogeny and DNA barcoding: a case study on Fritillaria. Scientific Reports 8:1184. DOI 10.1038/s41598-018-19591-9

Bolger AM, Lohse M, and Usadel B. 2014. Trimmomatic: a flexible trimmer for Illumina sequence data. Bioinformatics 30:2114-2120. DOI 10.1093/bioinformatics/btu170

Burke SV, Wysocki WP, Zuloaga FO, Craine JM, Pires JC, Edger PP, Mayfieldjones D, Clark LG, Kelchner SA, and Duvall MR. 2016. Evolutionary relationships in Panicoid grasses based on plastome phylogenomics

Peer] reviewing PDF | (2019:04:36376:1:2:NEW 25 Jun 2019) 
(Panicoideae; Poaceae). BMC Plant Biology 16:140. DOI 10.1186/s12870-016-0823-3

Byng JW, Chase MW, Christenhusz MJ, Fay MF, Judd WS, Mabberley DJ, Sennikov AN, Soltis DE, Soltis PS, and Stevens PF. 2016. An update of the Angiosperm Phylogeny Group classification for the orders and families of flowering plants: APG IV. Botanical Journal of the Linnean Society 181:1-20. DOI 10.1111/boj.12385

Carbonell-caballero J, Alonso R, Ibañez V, Terol J, Talon M, and Dopazo J. 2015. A phylogenetic analysis of 34 chloroplast genomes elucidates the relationships between wild and domestic species within the genus Citrus. Molecular Biology and Evolution 32:2015. DOI 10.1093/molbev/msv082

Cavalier-Smith T. 2002. Chloroplast evolution: secondary symbiogenesis and multiple losses. Current Biology Cb 12:R62-R64. DOI 10.1016/S0960-9822(01)00675-3

Çelebi A, Tekşen M, Açik L, and Aytaç Z. 2008. Taxonomic relationships in genus Fritillaria (Liliaceae): Evidence from RAPD-PCR and SDS-PAGE of seed proteins. Acta Botanica Hungarica 50:325-343. DOI 10.1556/ABot.50.2008.3-4.11

Chen SC, and Helen VM. 2000. Fritillaria L. In: Wu Z.Y \& Raven PH (Eds.) Flora of China (24th). Beijing: Science Press.

Chen XL, Zhou JG, Cui YX, Wang Y, Duan BZ, and Yao H. 2018. Identification of Ligularia herbs using the complete chloroplast genome as a super-barcode. Frontiers in Pharmacology 9:695. DOI 10.3389/fphar.2018.00695

Cosner ME, Jansen RK, Palmer JD, and Downie SR. 1997. The highly rearranged chloroplast genome of Trachelium caeruleum (Campanulaceae): multiple inversions, inverted repeat expansion and contraction, transposition, insertions/deletions, and several repeat families. Current Genetics 31:419-429.

Dang YY, Yang Y, Li Q, Lu JJ, Li XW, and Wang YT. 2014. Complete chloroplast genome sequences of poisonous and medicinal plant Datura stramonium: organizations and implications for genetic engineering. Plos One 9:e110656. DOI 10.1371/journal.pone.0110656

Darriba D, Taboada GL, Doallo R, and Posada D. 2012. jModelTest 2: more models, new heuristics and highperformance computing. Nature Methods 9:772. DOI 10.1038/nmeth.2109

Day PD, Madeleine B, Laurence H, Fay MF, Leitch AR, Leitch IJ, and Kelly LJ. 2014. Evolutionary relationships in the medicinally important genus Fritillaria L. (Liliaceae). Molecular Phylogenetics and Evolution 80:11-19. DOI 10.1016/j.ympev.2014.07.024

Ding YQ, Fang Y, Guo L, Li ZD, He KZ, Zhao Y, and Zhao H. 2017. Phylogenic study of Lemnoideae (duckweeds) through complete chloroplast genomes for eight accessions. Peer $J$ 5:e4186. DOI 10.7717 peerj. 4186

Do HDK, Kim JS, and Kim JH. 2014. A trnL_CAU triplication event in the complete chloroplast genome of Paris verticillata M.Bieb. (Melanthiaceae, Liliales). Genome Biology and Evolution 6:1699-1706. DOI 10.1093/gbe/evu138

Doyle J. 1987. A rapid DNA isolation procedure for small quantities of fresh leaf tissue. Phytochem Bull 19:11-15.

Du YP, Bi Y, Yang FP, Zhang MF, Chen XQ, Xue J, and Zhang XH. 2017. Complete chloroplast genome sequences of Lilium: insights into evolutionary dynamics and phylogenetic analyses. Scientific Reports 7:5751. DOI 10.1038/s41598-017-06210-2

Frazer KA, Pachter L, Poliakov A, Rubin EM, and Dubchak I. 2004. VISTA: computational tools for comparative genomics. Nucleic Acids Research 32:W273-W279. DOI 10.1093/nar/gkh458 
612 Gao L, Yi X, Yang YX, Su YJ, and Wang T. 2009. Complete chloroplast genome sequence of a tree fern

613

614

615

616

617

618

619

620

621

622

623

624

625

626

627

628

629

630

631

632

633

634

635

636

637

638

639

640

641

642

643

644

645

646

647

648

649

650

651

652 Alsophila spinulosa: insights into evolutionary changes in fern chloroplast genomes. BMC Evolutionary Biology 9:130. DOI 10.1186/1471-2148-9-130

Gao T, Hui Y, Song J, Zhu Y, Chang L, and Chen S. 2010. Evaluating the feasibility of using candidate DNA barcodes in discriminating species of the large Asteraceae family. BMC Evolutionary Biology 10:324. DOI 10.1186/1471-2148-10-324

Goremykin VV, Hirschernst KI, Wolfl S, and Hellwig FH. 2003. Analysis of the Amborella trichopoda chloroplast genome sequence suggests that Amborella is not a basal angiosperm. Molecular Biology and Evolution 20:1499-1505. DOI 10.1093/molbev/msg159

Goremykin VV, Hirschernst KI, Wölfl S, and Hellwig FH. 2004. The chloroplast genome of Nymphaea alba: whole-genome analyses and the problem of identifying the most basal angiosperm. Molecular Biology and Evolution 21:1445-1454. DOI 10.1093/molbev/msh147

Hall TA. 1999. BioEdit : a user-friendly biological sequence alignment editor and analysis program for Windows 95/98/NT. Nucleic Acids Symposium Series 41:95-98.

Henriquez CL, Arias T, Pires JC, Croat TB, and Schaal BA. 2014. Phylogenomics of the plant family Araceae. Molecular Phylogenetics \& Evolution 75:91. DOI 10.1016/j.ympev.2014.02.017

Huang J, Yang LQ, Yu Y, Liu YM, Xie DF, Li J, He XJ, and Zhou SD. 2018. Molecular phylogenetics and historical biogeography of the tribe Lilieae (Liliaceae): bi-directional dispersal between biodiversity hotspots in Eurasia. Annals of Botany:1-18. DOI 10.1093/aob/mcy138

Jin JJ, Yu WB, Yang JB, Song Y, Yi TS, and Li DZ. 2018. GetOrganelle: a simple and fast pipeline for de novo assembly of a complete circular chloroplast genome using genome skimming data. bioRxiv:1-11. DOI $10.1101 / 256479$

Katoh K, and Standley D. 2013. MAFFT multiple sequence alignment software version improvements in performance and usability. Molecular Biology \& Evolution 30:772-780. DOI 10.1093/molbev/mst010

Kearse M, Moir R, Wilson A, Stoneshavas S, Cheung M, Sturrock S, Buxton S, Cooper A, Markowitz S, and Duran C. 2012. Geneious Basic: An integrated and extendable desktop software platform for the organization and analysis of sequence data. Bioinformatics 28:1647-1649. DOI 10.1093/bioinformatics/bts 199

Khourang M, Babaei A, Sefidkon F, Naghavi MR, Asgari D, and Potter D. 2014. Phylogenetic relationship in Fritillaria spp. of Iran inferred from ribosomal ITS and chloroplast trnL-trnF sequence data. Biochemical Systematics \& Ecology 57:451-457. DOI 10.1016/j.bse.2014.10.001

Kress WJ, Wurdack KJ, Zimmer EA, Weigt LA, and Janzen DH. 2005. Use of DNA barcodes to identify flowering plants. Proceedings of the National Academy of Sciences of the United States of America 102:83698374. DOI 10.1073/pnas.0503123102

Kumar S, Stecher G, and Tamura K. 2016. MEGA7: molecular evolutionary genetics analysis version 7.0 for bigger datasets. Molecular Biology \& Evolution 33:1870-1874. DOI 10.1093/molbev/msw054

Kurtz S, Choudhuri JV, Ohlebusch E, Schleiermacher C, Stoye J, and Giegerich R. 2001. REPuter: the manifold applications of repeat analysis on a genomic scale. Nucleic Acids Research 29:4633-4642. DOI $10.1093 / \mathrm{nar} / 29.22 .4633$

Li PB, Li ZH, Liu HM, and Hua JP. 2014a. Cytoplasmic diversity of the cotton genus as revealed by chloroplast microsatellite markers. Genetic Resources \& Crop Evolution 61:107-119. DOI 10.1007/s10722-013-0018-9

Li QS, Li Y, Song JY, Xu HB, Xu J, Zhu YJ, Li XW, Gao HH, Dong LL, Qian J, Sun c, and Chen SI. $2014 b$.

Peer) reviewing PDF | (2019:04:36376:1:2:NEW 25 Jun 2019) 
653

654

655

656

657

658

659

660

661

662

663

664

665

666

667

668

669

670

671

672

673

674

675

676

677

678

679

680

681

682

683

684

685

686

687

688

689

690

691

692

693

High-accuracy de novo assembly and SNP detection of chloroplast genomes using a SMRT circular consensus sequencing strategy. New Phytologist 204:1041-1049. DOI 10.1111/nph.12966

Li Y, Li QS, Li XW, Song JY, and Sun C. 2016. Complete chloroplast genome sequence of Fritillaria unibracteata var. wabuensis based on SMRT sequencing technology. Mitochondrial DNA 27:3757-3758. DOI 10.3109/19401736.2015.1079892

Li Y, Zhang ZR, Yang JB, and Lv GH. 2018. Complete chloroplast genome of seven Fritillaria species, variable DNA markers identification and phylogenetic relationships within the genus. Plos One 13:e0194613. DOI 10.1371/journal.pone.0194613

Liu QP, and Xue QZ. 2005. Comparative studies on codon usage pattern of chloroplasts and their host nuclear genes in four plant species. Journal of Genetics 84:55-62. DOI 10.1007/bf02715890

Liu ZD, Wang S, and Chen SC. 2009. A taxonomic note of Fritillaria wabuensis (Liliaceae). Acta Botanica Yunnanica 31:145. DOI 10.3724/SP.J.1143.2009.09056

Liu ZF, Ci XQ, Li L, Li HW, Conra JG, and Li J. 2017. DNA barcoding evaluation and implications for phylogenetic relationships in Lauraceae from China. Plos One 12:e175788. DOI 10.1371/journal.pone.0175788

Lohse M, Drechsel O, Kahlau S, and Bock R. 2013. OrganellarGenomeDRAW - a suite of tools for generating physical maps of plastid and mitochondrial genomes and visualizing expression data sets. Nucleic Acids Research 41:W575-W581. DOI 10.1093/nar/gkt289

Lu RS, Li P, and Qiu YX. 2016. The complete chloroplast genomes of three Cardiocrinum (Liliaceae) species: comparative genomic and phylogenetic analyses. Frontiers in Plant Science 7:2054. DOI 10.3389/fpls.2016.02054(2016)

Luo YB, and Chen SC. 1996. A revision of Fritillaria L. (Liliaceae) in the hengduan mountains and adjacent regions, China(1) - A study of Fritillaria cirrhosa D. Don and its related species. Acta Phytotaxona Sin 34:304-312.

Ma PF, Zhang YX, Zeng CX, Guo ZH, and Li DZ. 2014. Chloroplast phylogenomic analyses resolve deep-level relationships of an intractable bamboo tribe Arundinarieae (poaceae). Systematic Biology 63:933-950. DOI 10.1093/sysbio/syu054

Ma SJ, Zhou JG, Li Y, Chen XL, Wu ML, Sun W, Li YH, Song JY, and Yao H. 2018. Complete chloroplast genomes of Dioscorea opposite and D. collettii and screening specific DNA barcodes (in Chinese). Scientia Sinica Vitae 48:571-582. DOI 10.1360/N052017-00160

Maliga P. 2002. Engineering the plastid genome of higher plants. Current Opinion in Plant Biology 5:164-172. DOI $10.1016 / \mathrm{S} 1369-5266(02) 00248-0$

Mardis ER. 2008. The impact of next-generation sequencing technology on genetics. Trends in Genetics 24:133141. DOI 10.1016/j.tig.2007.12.007

Mishra P, Kumar A, Nagireddy A, Mani DN, Shukla AK, Tiwari R, and Sundaresan V. 2015. DNA barcoding: an efficient tool to overcome authentication challenges in the herbal market. Plant Biotechnology Journal 14:821. DOI 10.1111/pbi.12419

Moore MJ, Soltis PS, Bell CD, Burleigh JG, and Soltis DE. 2010. Phylogenetic analysis of 83 plastid genes further resolves the early diversification of eudicots. Proceedings of the National Academy of Sciences of the United States of America 107:4623-4628. DOI 10.1073/pnas.0907801107

Murat C, Riccioni C, Belfiori B, Cichocki N, Labbe J, Morin E, Tisserant E, Paolocci F, Rubini A, and 
694

695

696

697

698

699

700

701

702

703

704

705

706

707

708

709

710

711

712

713

714

715

716

717

718

719

720

721

722

723

724

725

726

727

728

729

730

731

732

733

734

Martin F. 2011. Distribution and localization of microsatellites in the Perigord black truffle genome and identification of new molecular markers. Fungal Genetic Biology 48:592-601. DOI 10.1016/j.fgb.2010.10.007

National Pharmacopoeia Committee. 2015. Pharmacopoeia of the People's Republic of China, 2015ed. Beijing, China: Chemical Industry Press.

Neuhaus HE, and Emes MJ. 2000. Nonphotosynthetic metabolism in plastids. Annual Review Plant Physiology and Plant Molecular Biology 51:111-140. DOI 10.1146/annurev.arplant.51.1.111

Nie XJ, Lv SZ, Zhang YX, Du XH, Wang L, Biradar SS, Tan XF, Wan FH, and Song WN. 2012. Complete chloroplast genome sequence of a major invasive species, crofton weed (Ageratina adenophora). Plos One 7:e36869. DOI 10.1371/journal.pone.0036869

Ohyama K, Fukuzawa H, Kohchi T, Shirai H, Sano T, Sano S, Umesono K, Shiki Y, Takeuchi M, and Chang Z. 1986. Chloroplast gene organization deduced from complete sequence of liverwort Marchantia polymorpha chloroplast DNA. Nature 322:572-574. DOI 10.1038/322572a0

Park I, Kim WJ, Yeo SM, Choi G, Kang YM, Piao RZ, and Moon BC. 2017. The complete chloroplast genome sequences of Fritillaria ussuriensis Maxim. and Fritillaria cirrhosa D. Don, and comparative analysis with other Fritillaria species. Molecules 22:982. DOI 10.3390/molecules22060982

Parks M, Cronn R, and Liston A. 2009. Increasing phylogenetic resolution at low taxonomic levels using massively parallel sequencing of chloroplast genomes. BMC Biology 7:84. DOI 10.1186/1741-7007-7-84

Pauwels M, Vekemans X, Godé C, Frérot H, Castric V, and Saumitoulaprade P. 2012. Nuclear and chloroplast DNA phylogeography reveals vicariance among European populations of the model species for the study of metal tolerance, Arabidopsis halleri (Brassicaceae). New Phytologist 193:916-928. DOI 10.1111/j.14698137.2011.04003.x

Percy DM, Argus GW, Cronk QC, Fazekas AJ, Kesanakurti PR, Burgess KS, Husband BC, Newmaster SG, Barrett SC, and Graham SW. 2014. Understanding the spectacular failure of DNA barcoding in willows (Salix): does this result from a trans-specific selective sweep? Molecular Ecology 23:4737-4756. DOI 10.1111/mec.12837

Powell W, Morgante M, Mcdevitt R, Vendramin GG, and Rafalski JA. 1995. Polymorphic simple sequence repeat regions in chloroplast genomes: applications to the population genetics of pines. Proceedings of the National Academy of Sciences of the United States of America 92:7759. DOI 10.1073/pnas.92.17.7759

Qian J, Song JY, Gao HH, Zhu YJ, Xu J, Pang XH, Yao H, Sun C, Li XE, and Li CY. 2013. The complete chloroplast genome sequence of the medicinal plant Salvia miltiorrhiza. Plos One 8:e57607. DOI 10.1371/journal.pone.0057607

Raubeson AL, Peery R, Chumley TW, Chris D, Matthew FH, Boore JL, and Jansen RK. 2007. Comparative chloroplast genomics: analyses including new sequences from the angiosperms Nuphar advena and Ranunculus macranthus. BMC Genomics 8:174. DOI 10.1186/1471-2164-8-174

Rix M, Frank E, Webster G, and Group AGSF. 2001. Fritillaria: A Revised Classification : Together with an Updated List of Species: Fritillaria Group of the Alpine Garden Society, UK.

Ronquist F, Teslenko M, Van dMP, Ayres DL, Darling A, Höhna S, Larget B, Liu L, Suchard MA, and Huelsenbeck JP. 2012. MrBayes 3.2: efficient Bayesian phylogenetic inference and model choice across a large model space. Systematic Biology 61:539-542. DOI 10.1093/sysbio/sys029

Rønsted N, Law S, Thornton H, Fay MF, and Chase MW. 2005. Molecular phylogenetic evidence for the monophyly of Fritillaria and Lilium (Liliaceae; Liliales) and the infrageneric classification of Fritillaria.

Peer) reviewing PDF | (2019:04:36376:1:2:NEW 25 Jun 2019) 
Molecular Phylogenetics and Evolution 35:509-527. DOI 10.1016/j.ympev.2004.12.023

Rose O, and Falush D. 1998. A threshold size for microsatellite expansion. Molecular Biology and Evolution 15:613-615. DOI 10.1093/oxfordjournals.molbev.a025964

Rozas J, Ferrermata A, Sánchezdelbarrio JC, Guiraorico S, Librado P, Ramosonsins SE, and Sánchezgracia A. 2017. DnaSP 6: DNA sequence polymorphism analysis of large datasets. Molecular Biology and Evolution 34:3299-3302. DOI 10.1093/molbev/msx248

Shendure J, and Ji H. 2008. Next-generation DNA sequencing. Nature Biotechnology 26:1135-1145. DOI $10.1038 / \mathrm{nbt} 1486$

Shinozaki K, Ohme M, Tanaka M, Wakasugi T, Hayashida N, Matsubayashi T, Zaita N, Chunwongse J, Obokata J, and Yamaguchishinozaki K. 1986. The complete nucleotide sequence of the tobacco chloroplast genome: its gene organization and expression. Plant Molecular Biology Reporter 5:2043-2049.

Simmons MP, Zhang LB, Webb CT, and Müller K. 2007. A penalty of using anonymous dominant markers (AFLPs, ISSRs, and RAPDs) for phylogenetic inference. Molecular Phylogenetics and Evolution 42:528-542. DOI 10.1016/j.ympev.2006.08.008

Stamatakis A. 2014. RAxML version 8: a tool for phylogenetic analysis and post-analysis of large phylogenies. Bioinformatics 30:1312-1313. DOI 10.1093/bioinformatics/btu033

Sugiura M. 1992. The chloroplast genome. Plant Molecular Biology 19:149-157.

Tangphatsornruang S, Sangsrakru D, Chanprasert J, Uthaipaisanwong P, Yoocha T, Jomchai N, and Tragoonrung S. 2009. The chloroplast genome sequence of Mungbean (Vigna radiata) determined by Highthroughput pyrosequencing: structural organization and phylogenetic relationships. DNA Research 17:11. DOI 10.1093/dnares/dsp025(2010)

Tekşen M, Aytaç Z, and Pınar NM. 2010. Pollen morphology of the genus Fritillaria L. (Liliaceae) in Turkey. Turkish Journal of Botany 34:397-416. DOI 10.3906/bot-0907-93

The angiosperm phylogeny group. 1998. An ordinal classification for the families of flowering plants. Annals of the Missouri botanical Garden:531-553. DOI 10.2307/2992015

The angiosperm phylogeny group. 2003. An update of the Angiosperm Phylogeny Group classification for the orders and families of flowering plants: APG II. Botanical Journal of the Linnean Society 141:399-436. DOI 10.1046/j.1095-8339.2003.t01-1-00158.x

The angiosperm phylogeny group. 2009. An update of the Angiosperm Phylogeny Group classification for the orders and families of flowering plants: APG III. Botanical Journal of the Linnean Society 161:105-121. DOI 10.1111/j.1095-8339.2009.00996.x

Vieira LDN, Faoro H, Rogalski M, Fraga HPDF, Cardoso RLA, Souza EMD, Pedrosa FDO, Nodari RO, and Guerra MP. 2014. The complete chloroplast genome sequence of podocarpus lambertii: genome structure, evolutionary aspects, gene content and SSR detection. Plos One 9:e90618. DOI 10.1371/journal.pone.0090618

Wang MX, Cui LC, Feng KW, Deng PC, Du XH, Wan FH, Song WN, and Nie XJ. 2015. Comparative analysis of Asteraceae chloroplast genomes: structural organization, RNA editing and evolution. Plant Molecular Biology Reporter 33:1526-1538. DOI 10.1007/s11105-015-0853-2

Wang RJ, Cheng CL, Chang CC, Wu CL, Su TM, and Chaw SM. 2008. Dynamics and evolution of the inverted repeat-large single copy junctions in the chloroplast genomes of monocots.(Research article). BMC Evolutionary Biology 8:36. DOI 10.1186/1471-2148-8-36

Wick RR, Schultz MB, Zobel J, and Holt KE. 2015. Bandage: interactive visualization of de novo genome 
assemblies. Bioinformatics 31:3350-3352. DOI 10.1093/bioinformatics/btv383

Wietsma WA, Deinum D, Teunissen HAS, and Berg RGVD. 2015. Phylogenetic relationships within Fritillaria section Petilium based on AFLP fingerprints. Plant Systematics and Evolution 301:1043-1054. DOI 10.1007/s00606-014-1135-4

Wu FH, Chan MT, Liao DC, Hsu CT, Lee YW, Daniell H, Duvall MR, and Lin CS. 2010. Complete chloroplast genome of Oncidium Gower Ramsey and evaluation of molecular markers for identification and breeding in Oncidiinae. BMC Plant Biology 10:68. DOI 10.1186/1471-2229-10-68

Xue JH, Dong WP, Cheng T, and Zhou SL. 2012. Nelumbonaceae: Systematic position and species diversification revealed by the complete chloroplast genome. Journal of Systematics and Evolution 50:477-487. DOI 10.1111/j.1759-6831.2012.00224.x

Yang JB, Li DZ, and Li HT. 2014. Highly effective sequencing whole chloroplast genomes of angiosperms by nine novel universal primer pairs. Molecular Ecology Resources 14:1024-1031. DOI 10.1111/1755-0998.12251

Yi X, Gao L, Wang B, Su YJ, and Wang T. 2013. The complete chloroplast genome sequence of cephalotaxus oliveri (Cephalotaxaceae): Evolutionary comparison of cephalotaxus chloroplast DNAs and insights into the loss of inverted repeat copies in Gymnosperms. Genome Biology and Evolution 5:688-698. DOI $10.1093 /$ gbe/evt042

Yuan C, Zhong W, Mou F, Gong Y, Pu D, Ji P, Huang H, Yang Z, and Zhang C. 2017. The complete chloroplast genome sequence and phylogenetic analysis of Chuanminshen (Chuanminshenviolaceum Sheh et Shan). Physiology \& Molecular Biology of Plants 23:1-7. DOI 10.1007/s12298-016-0395-6

Yurina NP, and Odintsova MS. 1998. Compsrative structural organization of plant chloroplast and mitochondrial genomes. Genetika 34:5-22.

Zhang DQ, Mo XC, Xiang JY, and Zhou N. 2016. Molecular identification of original plants of Fritillariae cirrhosae bulbus, a tradtional chinese medicine (TCM) using plant dna barcoding. African Journal of Traditional Complementary \& Alternative Medicines Ajtcam 13:74. DOI 10.21010/ajtcam.v13i6.12

Zhang LN, Zhang YJ, and SUN jZ. 2001. A taxometric analysis of the variation patterns of characters of Fritillaria przewalskii ( Liliaceae). Acta Bot Boreal-Occident Sin 21:844-856.

Zhang YJ, and Cheng L. 1998. Taxonomic Studies of Fritillaria ( Liliaceae) from Gansu Province. Journal of Lanzhou University (Natural Sciences) 32:84-91. DOI 10.13885/j.issn.0455-2059.1998.02.017 
804 Table 1:

805 Summary of complete chloroplast genomes for eight Fritillaria species

806

807 Table 2:

808 Base composition in Fritillaria cirrhosa chloroplast genome

809

810 Table 3:

811 Gene contents in eight Fritillaria chloroplast genome

812 Notes: The I label after gene names reflect genes located in IR regions. Intron containing gene is 813 indicated by one asterisk.

815 Table 4:

816 Number of nucleotide substitutions and sequence distance in eight complete chloroplast genomes

817 Notes: The upper triangle shows number of nucleotide substitutions and the lower triangle 818 indicates genetic distance in complete cp genomes among species.

819

820

Table 5:

821 Variable site analysis in Fritillaria chloroplast genomes 
829 Figure 1. Distribution of Fritillaria cirrhosa and its closely related species. The distribution area 830 of each species is drawn according to the records of Luo et al. (1996), Liu et al. (2009) and some 831 existing voucher specimens (http://www.cvh.ac.cn/). Photos of representative living plants of 832 eight Fritillaria species: A. F. cirrhosa, B. F. sichuanica, C. F. taipaiensis, D. F. yuzhongensis, 833 E. F. unibracteata, F. F. przewalskii, G. F. sinica, H. F. dajinensis. Topographic data digital 834 elevation modeling (DEM) data were required from the USGS website 835 (https://glovis.usgs.gov/app?tour) with a 90-m spatial resolution grid.

Figure 2. Gene map of Fritillaria chloroplast genomes. Genes outside the circle are transcribed clockwise, and genes shown on the inside of the circle are counter-clockwise. Genes belonging to functional group are color-coded. The darker gray in the inner corresponds to GC content, and the lighter gray corresponds to AT content.

Figure 3. Analysis of simple sequence repeat (SSR) in eight Fritillaria cp genomes. A. Number different SSRs type detected in nine genomes; B. Frequency of SSR motifs in different repeat types of F. cirrhosa cp genome; C. Frequency of identified SSR in LSC, SSC, and IR regions; D. Frequency of identified SSR in IGS, CDS, and intron.

Figure 4. Analysis of large repeat sequences in eight Fritillaria cp genomes. A. Total of five repeat types; B. Frequency of tandem repeats in IGS, CDS, and intron; C. Frequency of tandem repeats by length; D. Frequency of palindromic repeats by length; E. Frequency of forward repeats by length; F. Frequency of reverse repeats by length. 
854 Figure 5. Visualization alignment of nine Fritillaria $\mathrm{cp}$ genomes. VISTA-based identify plot 855 showing sequence identify among eight Fritillaria species using Fritillaria cirrhosa D. Don as a 856 reference. The thick black line shows the inverted repeats (IRs) in the chloroplast genomes.

857

858

860

861

862

863

864 865

866

867

868

869

870

871

872

873

874

Figure 6. Comparison of LSC, SSC, and IR border regions among eight Fritillaria $\mathrm{cp}$ genomes. Colored boxes for genes represent the gene position.

Figure 7. Sliding window analysis of eight Fritillaria cp genomes (window length: 600 bp, step size: 200 bp). X-axis: position of the midpoint of a window; Y-axis: nucleotide diversity of each window.

Figure 8. Phylogenetic relationship of nine Fritillaria species inferred from Bayesian analyses (BI), maximum parsimony (MP), and maximum likelihood (ML) of different datasets. A. Chloroplast genome (Only contains one IR); B. LSC region; C. SSC region; D. protein coding region. Number above nodes are support values with Bayesian posterior probabilities (PP) values on the left, MP bootstrap values in the middle, ML bootstrap values on the right.

1

3

4




\section{Figure 1}

Figure 1. Distribution of Fritillaria cirrhosa and its closely related species.

The distribution area of each species is drawn according to the records of Luo et al.(1996a,b), Liu et al. (2009) and some existing voucher specimens ( http://www.cvh.ac.cn /). Photos of representative living plants of eight Fritillaria species: A. F. cirrhosa, B. F. sichuanica, C. F. taipaiensis, D. F. yuzhongensis, E. F. unibracteata, F. F. przewalskii, G. F. sinica, H. F. dajinensis.

Topographic data digital elevation modeling (DEM) data were required from the USGS website ( https://glovis.usgs.gov/app?tour ) with a 90-m spatial resolution grid.

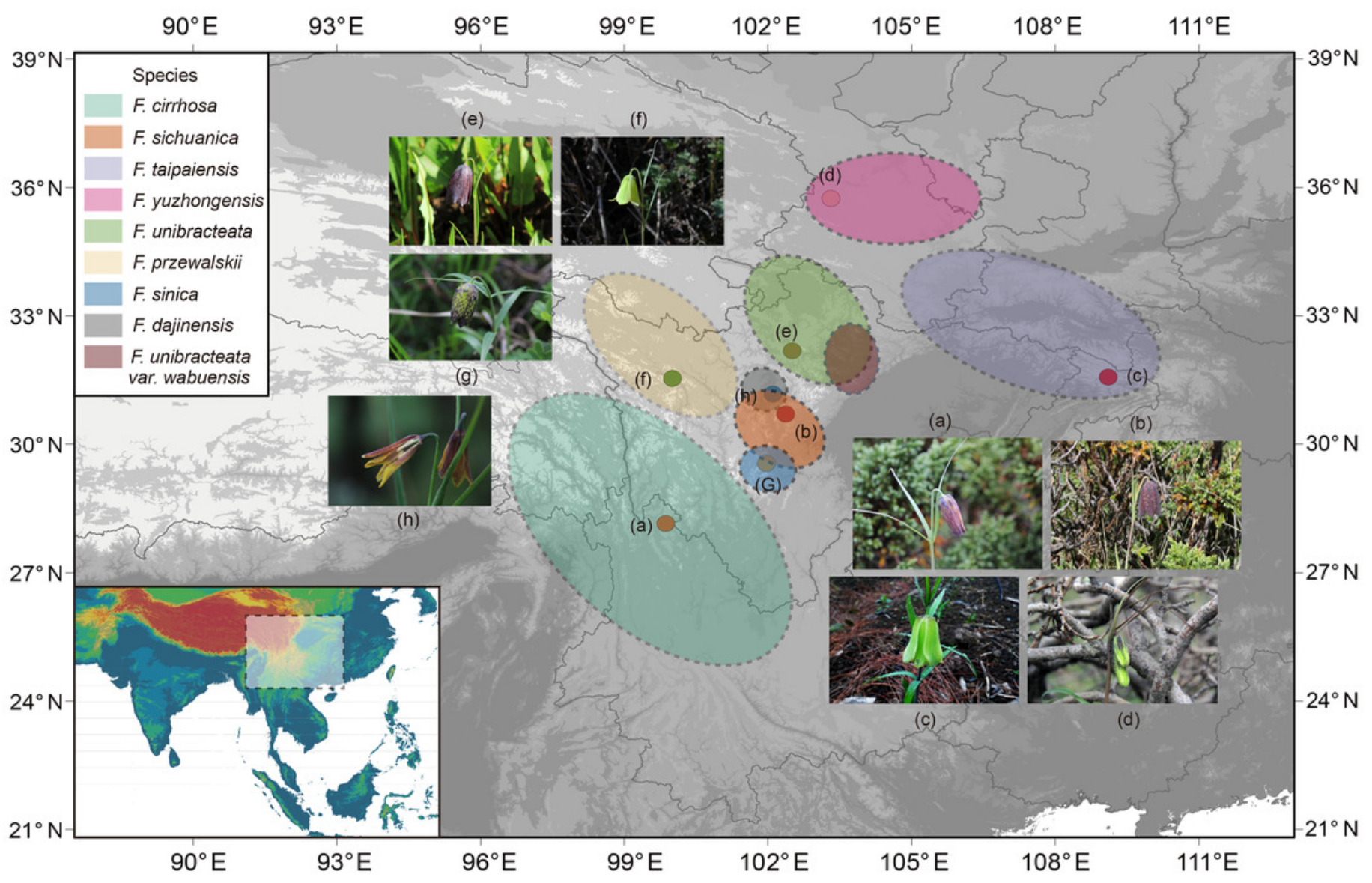


Figure 2 (on next page)

Gene map of Fritillaria chloroplast genomes.

Genes outside the circle are transcribed clockwise, and genes shown on the inside of the circle are counter-clockwise. Genes belonging to functional group are color-coded. The darker gray in the inner corresponds to GC content, and the lighter gray corresponds to AT content. 


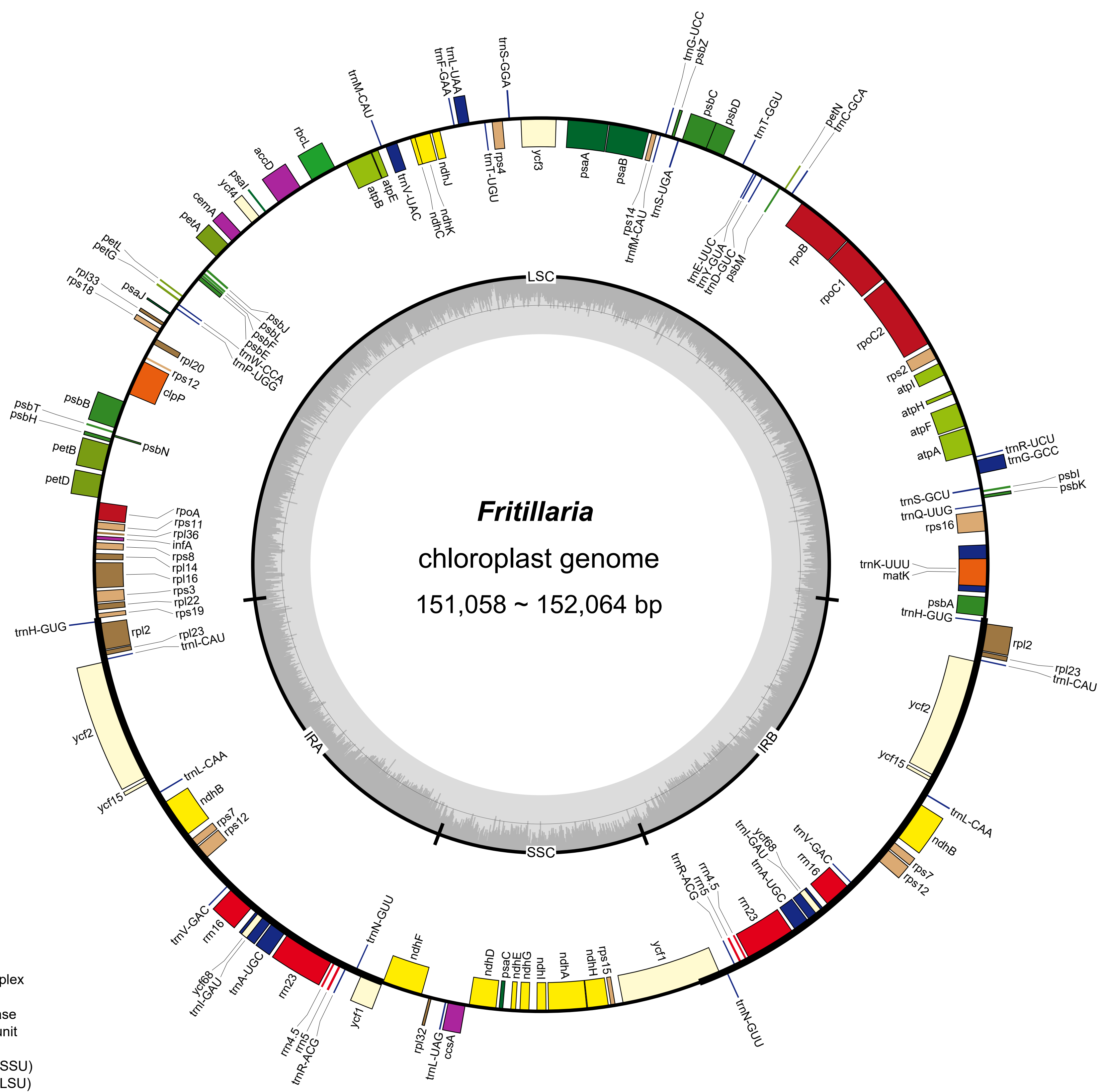

$\square$ ribosomal proteins (SSU)

$\square$ clpP, matK

$\square$ other genes

$\square$ hypothetical chloroplast reading frames (ycf) 
Figure 3 (on next page)

Analysis of simple sequence repeat (SSR) in eight Fritillaria cp genomes.

A. Number different SSRs type detected in nine genomes; B. Frequency of SSR motifs in different repeat types of $F$. cirrhosa cp genome; $\mathbf{C}$. Frequency of identified SSR in LSC, SSC, and IR regions; D. Frequency of identified SSR in IGS, CDS, and intron. 


\section{$\mathbf{A}$}

A

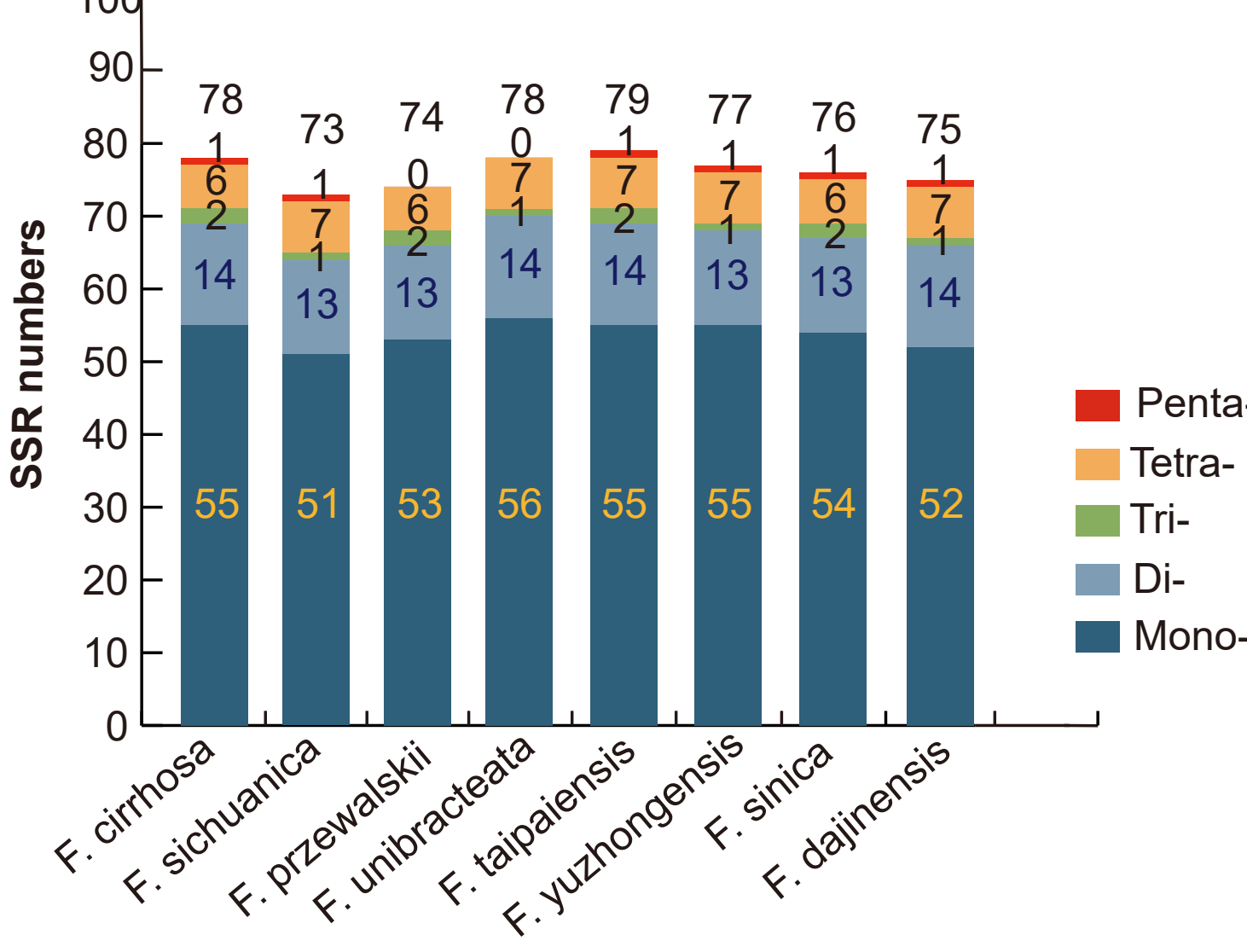

C

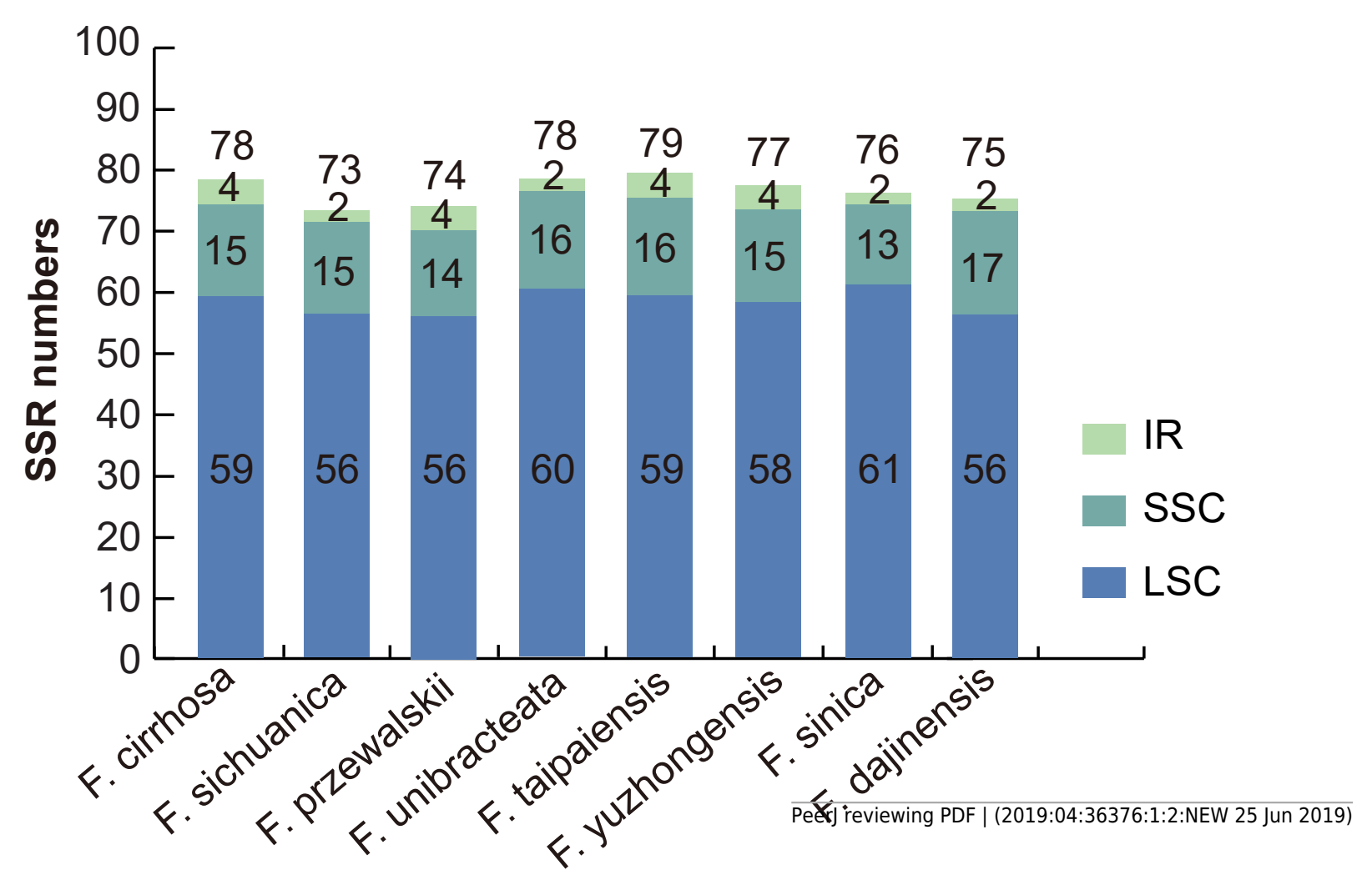

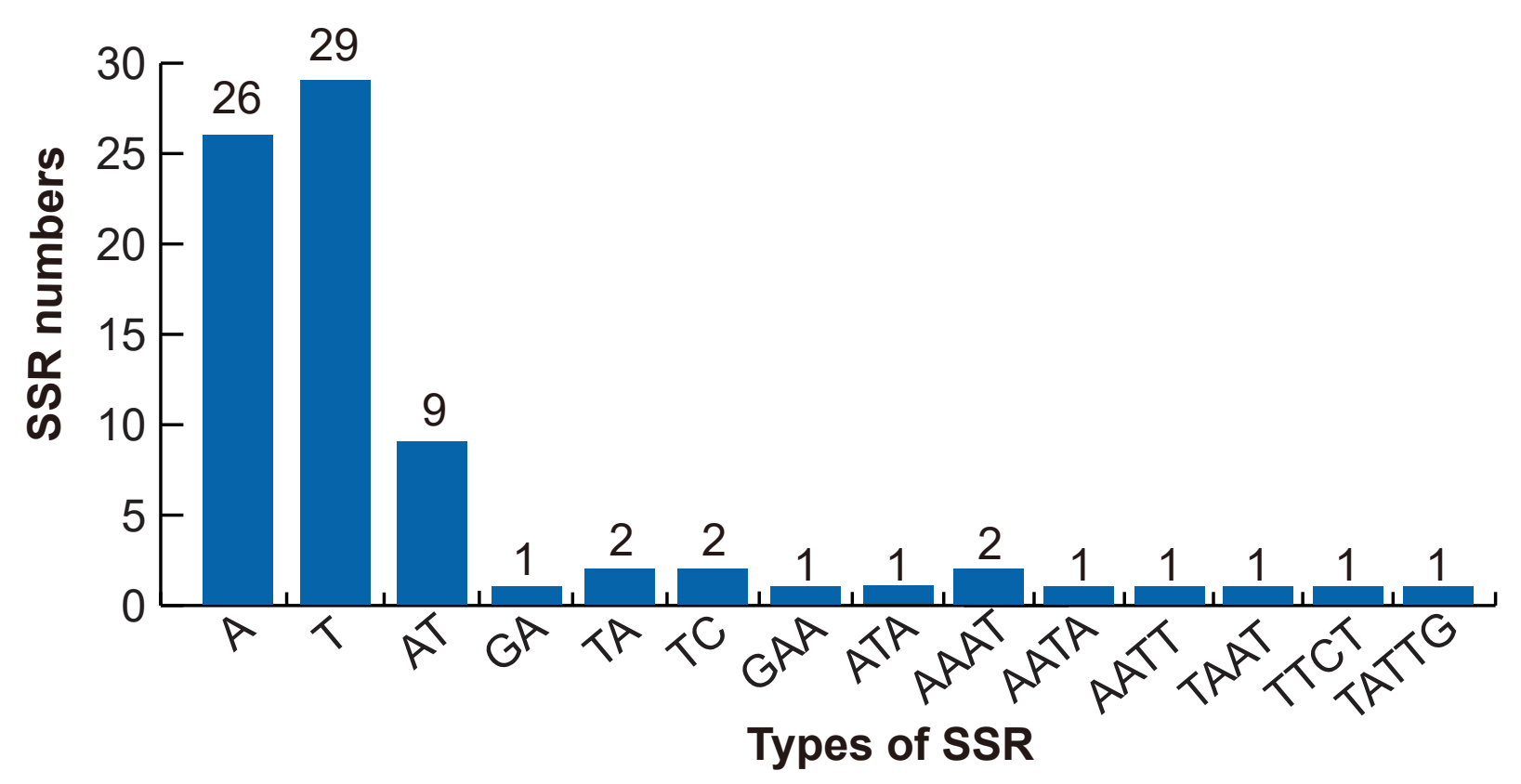

D

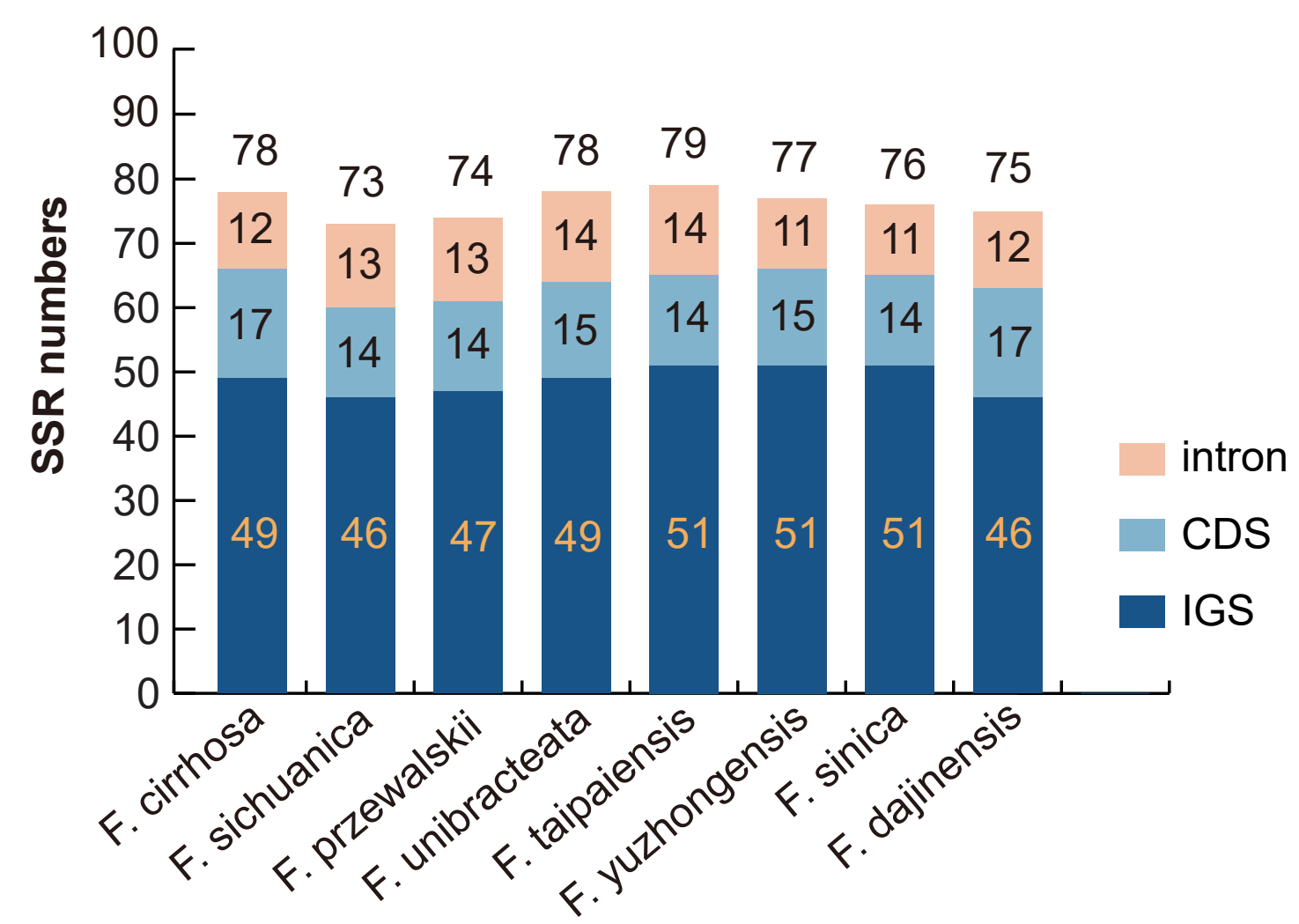


Figure 4 (on next page)

Analysis of large repeat sequences in eight Fritillaria cp genomes.

A. Total of five repeat types; B. Frequency of tandem repeats in IGS, CDS, and intron; C.

Frequency of tandem repeats by length; $\mathbf{D}$. Frequency of palindromic repeats by length; $\mathbf{E}$. Frequency of forward repeats by length; $\mathbf{F}$. Frequency of reverse repeats by length. 
Figure $\mathbf{5}$ (on next page)

Visualization alignment of nine Fritillaria cp genomes.

VISTA-based identify plot showing sequence identify among eight Fritillaria species using

Fritillaria cirrhosa D. Don as a reference. The thick black line shows the inverted repeats (IRs) in the chloroplast genomes. 
Figure 6 (on next page)

Comparison of LSC, SSC, and IR border regions among eight Fritillaria cp genomes.

Colored boxes for genes represent the gene position. 
Figure 7 (on next page)

Sliding window analysis of eight Fritillaria cp genomes (window length: $600 \mathrm{bp}$, step size: $200 \mathrm{bp}$ ).

X-axis: position of the midpoint of a window; Y-axis: nucleotide diversity of each window. 
IRa

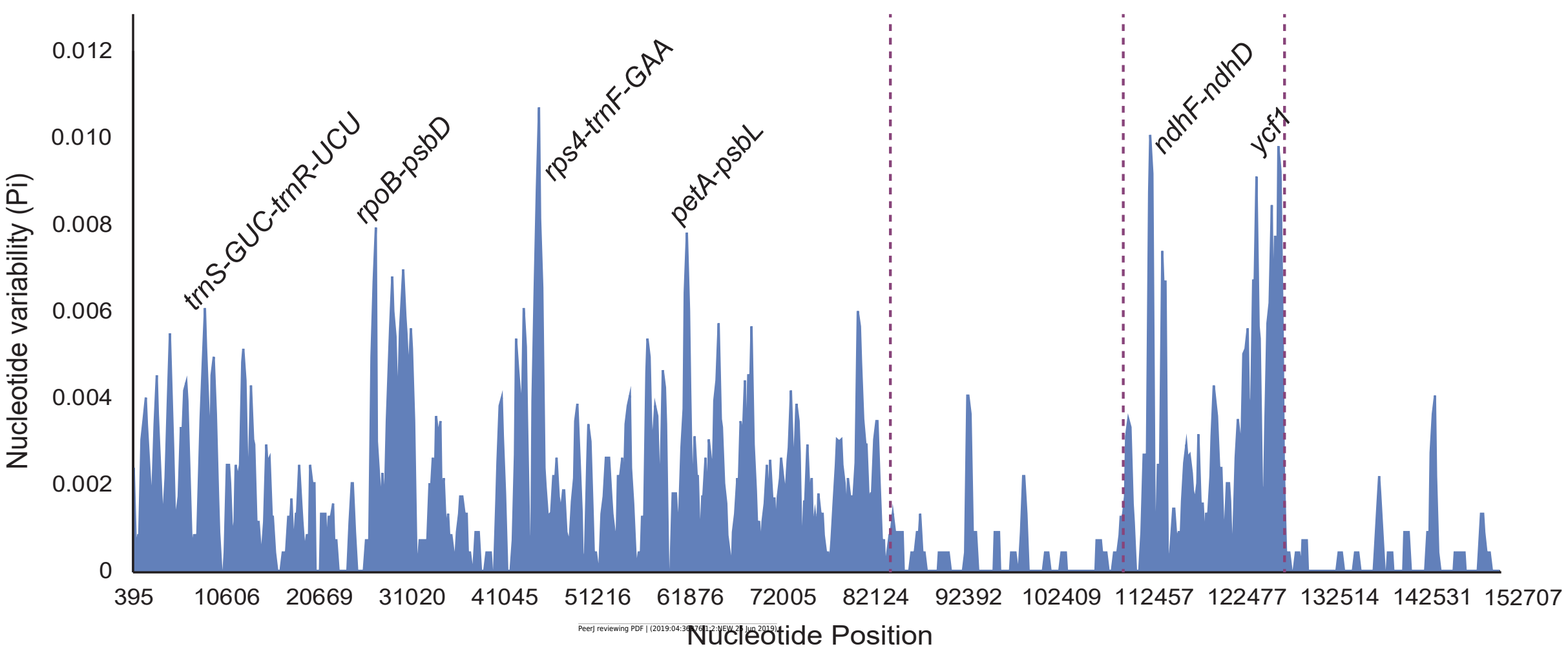


Figure 8 (on next page)

Phylogenetic relationship of nine Fritillaria species inferred from Bayesian analyses (BI), maximum parsimony (MP), and maximum likelihood (ML) of different datasets.
A. Chloroplast genome (Only contains one IR);
B. LSC region;
C. SSC region;
D. protein coding region. Number above nodes are support values with Bayesian posterior probabilities (PP) values on the left, MP bootstrap values in the middle, ML bootstrap values on the right. 


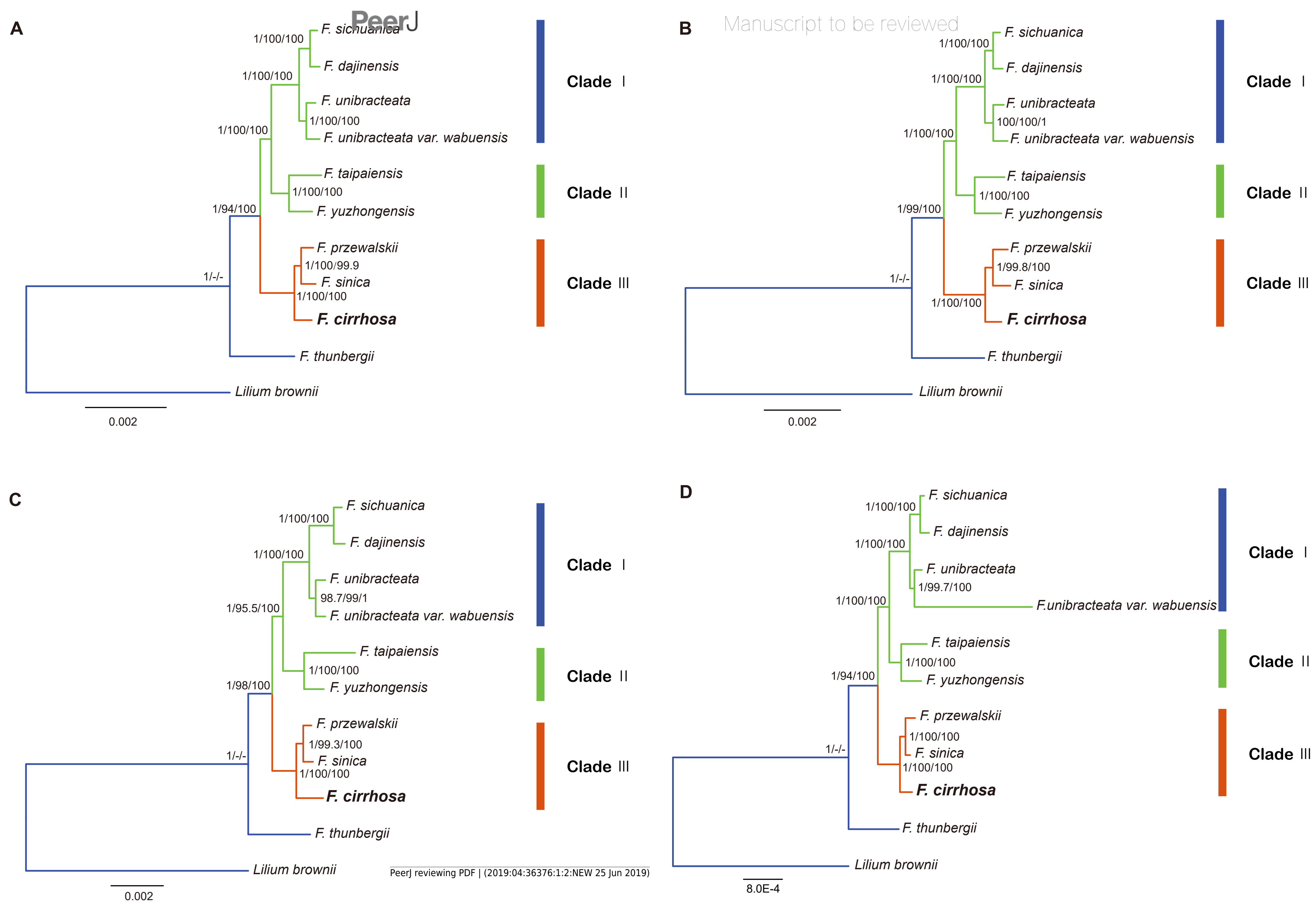




\section{Table 1 (on next page)}

Summary of complete chloroplast genomes for eight Fritillaria species 
Table 1:

2 Summary of complete chloroplast genomes for eight Fritillaria species

\begin{tabular}{|c|c|c|c|c|c|c|c|c|c|c|}
\hline & Total(bp) & $\begin{array}{l}\text { Large single } \\
\text { copy(LSC,bp) }\end{array}$ & $\begin{array}{l}\text { Small single } \\
\text { copy (SSC,bp) }\end{array}$ & $\begin{array}{l}\text { Inverted } \\
\text { repeat(IR,bp) }\end{array}$ & $\mathrm{GC} \%$ & $\begin{array}{l}\text { Total } \\
\text { genes }\end{array}$ & $\begin{array}{l}\text { Protein coding } \\
\text { genes }\end{array}$ & tRNA & rRNA & $\begin{array}{l}\text { Accession } \\
\text { number in } \\
\text { GenBank }\end{array}$ \\
\hline F. cirrhosa & 151,998 & 81,755 & 17,545 & 26,349 & $36.9 \%$ & 115 & 78 & 30 & 4 & MH244906 \\
\hline F. sichuanica & 151,958 & 81,726 & 17,542 & 26,345 & $37.0 \%$ & 115 & 78 & 30 & 4 & MH244907 \\
\hline F. przewalskii & 151,983 & 81,744 & 17,539 & 26,350 & $36.9 \%$ & 115 & 78 & 30 & 4 & MH244908 \\
\hline F. unibracteata & 151,058 & 81,339 & 17,539 & 26,090 & $37.0 \%$ & 115 & 78 & 30 & 4 & MH24490S \\
\hline F. taipaiensis & 151,707 & 81,451 & 17,552 & 26,352 & $37.0 \%$ & 115 & 78 & 30 & 4 & МH24491C \\
\hline F. yuzhongensis & 151,645 & 81,417 & 17,526 & 26,351 & $37.0 \%$ & 115 & 78 & 30 & 4 & MH244911 \\
\hline F. sinica & 152,064 & 81,827 & 17,537 & 26,350 & $36.9 \%$ & 115 & 78 & 30 & 4 & МH244912 \\
\hline F. dajinensis & 151,991 & 81,723 & 17,540 & 26,364 & $36.9 \%$ & 115 & 78 & 30 & 4 & MH244913 \\
\hline
\end{tabular}

3 
Table 2 (on next page)

Base composition in Fritillaria cirrhosa chloroplast genome 
1 Table 2:

2 Base composition in Fritillaria cirrhosa chloroplast genome

\begin{tabular}{lllllll}
\hline & $\mathrm{T} / \mathrm{U} \%$ & $\mathrm{C} \%$ & $\mathrm{~A} \%$ & $\mathrm{G} \%$ & $\mathrm{AT} \%$ & Length(bp) \\
\hline Genome & 31.9 & 18.8 & 31.1 & 18.1 & 63.1 & 151,998 \\
LSC & 33.3 & 17.9 & 31.9 & 17.0 & 65.1 & 81,755 \\
SSC & 35.0 & 16.1 & 34.5 & 14.4 & 69.5 & 17,545 \\
IR & 28.5 & 20.5 & 29 & 22.0 & 57.5 & 26,349 \\
tRNA & 25.0 & 23.7 & 21.9 & 29.4 & 46.9 & 2,877 \\
rRNA & 18.9 & 23.5 & 26.0 & 31.5 & 45.0 & 9,052 \\
Protein Coding genes & 31.7 & 17.3 & 31.0 & 20.0 & 62.7 & 68,234 \\
1st position codon & 24.6 & 18.1 & 30.9 & 26.4 & 55.5 & 22,745 \\
2nd position codon & 32.2 & 19.9 & 29.9 & 18.1 & 62.0 & 22,745 \\
3rd position codon & 38.3 & 14.0 & 32.1 & 15.6 & 70.4 & 22,744 \\
\hline
\end{tabular}

3 


\section{Table 3(on next page)}

Gene contents in eight Fritillaria chloroplast genome

The I label after gene names reflect genes located in IR regions. Intron containing gene is indicated by one asterisk 
Table 3:

2 Gene contents in eight Fritillaria chloroplast genome

\begin{tabular}{|c|c|c|}
\hline Category for gene & Group of genes & Name of genes \\
\hline \multirow[t]{5}{*}{ Self-replication } & Large subunit of ribosome & $r p l 2^{\mathrm{I}}, r p l 14, r p l 16^{*}, r p l 20, r p l 22, r p l 23^{\mathrm{I}}, r p l 32, r p l 33, r p l 36$ \\
\hline & Small subunit of ribosome & $r p s 2, r p s 3, r p s 4, r p s 7^{\mathrm{I}}, r p s 8, r p s 11, r p s 12^{1 *}, r p s 14, r p s 15, r p s 16^{*}, r p s 18, r p s 19$ \\
\hline & DNA dependent RNA polymerase & rpoA, rpoB, rpoC1*, rpoC2 \\
\hline & rRNA gene & $r r n 4.5^{\mathrm{I}}, r r n 5^{\mathrm{I}}, r r n 16^{\mathrm{I}}, r r n 23^{\mathrm{I}}$ \\
\hline & tRNA gene & 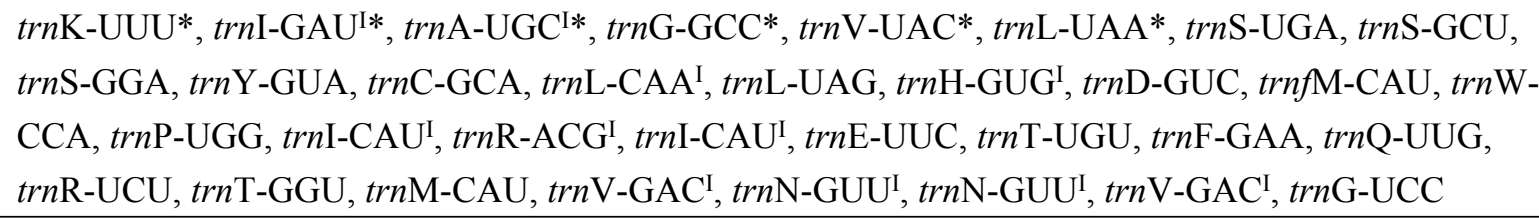 \\
\hline \multirow{6}{*}{$\begin{array}{l}\text { Gene for } \\
\text { photosynthesis }\end{array}$} & Subunits of photosystem I & $p s a \mathrm{~A}, p s a \mathrm{~B}, p s a \mathrm{C}, p s a \mathrm{I}, p s a \mathrm{~J}$ \\
\hline & Subunits of photosystem II & $p s b \mathrm{~A}, p s b \mathrm{~B}, p s b \mathrm{C}, p s b \mathrm{D}, p s b \mathrm{E}, p s b \mathrm{~F}, p s b \mathrm{H}, p s b \mathrm{I}, p s b \mathrm{~J}, p s b \mathrm{~K}, p s b \mathrm{~L}, p s b \mathrm{M}, p s b \mathrm{~N}, p s b \mathrm{~T}, p s b \mathrm{Z}$ \\
\hline & Subunits of NADH-dehydrogenase & $n d h \mathrm{~A}^{*}, n d h \mathrm{~B}^{\mathrm{I} *}, n d h \mathrm{C}, n d h \mathrm{D}, n d h \mathrm{E}, n d h \mathrm{~F}, n d h \mathrm{G}, n d h \mathrm{H}, n d h \mathrm{I}, n d h \mathrm{~J}, n d h \mathrm{~K}$ \\
\hline & Subunits of cytochrome $b / f$ complex & pet $\mathrm{A}, \operatorname{pet} \mathrm{B}^{*}, \operatorname{pet} \mathrm{D}^{*}, \operatorname{pet} \mathrm{G}, \operatorname{pet} \mathrm{L}, \operatorname{pet} \mathrm{N}$ \\
\hline & Subunit for ATP synthase & $a t p \mathrm{~A}, a t p \mathrm{~B}, a t p \mathrm{E}, a t p \mathrm{~F}^{*}, a t p \mathrm{H}, a t p \mathrm{I}$ \\
\hline & Large subunit of rubisco & $r b c \mathrm{~L}$ \\
\hline \multirow[t]{7}{*}{ Other genes } & Translational initiation factor & $\inf \mathrm{A}$ \\
\hline & Maturase & $\operatorname{mat} \mathrm{K}$ \\
\hline & Protease & $\operatorname{clp} \mathrm{P}^{*}$ \\
\hline & Envelope membrane protein & $\operatorname{cem} \mathrm{A}$ \\
\hline & Subunit of Acetyl-carboxylase & $a c c \mathrm{D}$ \\
\hline & C-type cytochrome synthesis gene & $\operatorname{ccs} \mathrm{A}$ \\
\hline & Open reading frames(ORF,ycf) & $y c f 1, y c f 2^{\mathrm{I}}, y c f 3^{*}, y c f 4, y c f 15^{\mathrm{I}}, y c f 68^{\mathrm{I}}$ \\
\hline
\end{tabular}

3 Notes.

4 The I label after gene names reflect genes located in IR regions. Intron containing gene is indicated by one asterisk. 


\section{Table 4(on next page)}

Number of nucleotide substitutions and sequence distance in eight complete chloroplast genomes

The upper triangle shows number of nucleotide substitutions and the lower triangle indicates genetic distance in complete cp genomes among species. 
1 Table 4:

2 Number of nucleotide substitutions and sequence distance in eight complete chloroplast genomes

\begin{tabular}{|c|c|c|c|c|c|c|c|c|}
\hline & $\begin{array}{l}F . \\
\text { cirrhosa }\end{array}$ & F. sichuanica & F. przewalskii & F. unibracteata & F. taipaiensis & $\begin{array}{l}F . \\
\text { yuzhongensis }\end{array}$ & F. sinica & F. dajinensis \\
\hline F. cirrhosa & & 311 & 112 & 314 & 335 & 310 & 117 & 311 \\
\hline F. sichuanica & 0.0021 & & 328 & 95 & 290 & 261 & 331 & 52 \\
\hline F. przewalskii & 0.0007 & 0.0022 & & 317 & 340 & 314 & 81 & 328 \\
\hline F. unibracteata & 0.0021 & 0.0006 & 0.0021 & & 277 & 252 & 320 & 105 \\
\hline F. taipaiensis & 0.0022 & 0.0019 & 0.0023 & 0.0018 & & 169 & 337 & 294 \\
\hline$F$ & 0.0021 & 0.0017 & 0.0021 & 0.0017 & 0.0011 & & 313 & 261 \\
\hline \multicolumn{9}{|l|}{ yuzhongensis } \\
\hline$F$. sinica & 0.0008 & 0.0022 & 0.0005 & 0.0021 & 0.0022 & 0.0021 & & 333 \\
\hline$F$. dajinensis & 0.0021 & 0.0003 & 0.0022 & 0.0007 & 0.0020 & 0.0017 & 0.0022 & \\
\hline
\end{tabular}

3 Notes.

4 The upper triangle shows number of nucleotide substitutions and the lower triangle indicates genetic distance in complete cp genomes 5 among species. 


\section{Table 5 (on next page)}

Variable site analysis in Fritillaria chloroplast genomes 
1 Table 5:

2 Variable site analysis in Fritillaria chloroplast genomes

\begin{tabular}{lllll}
\hline & Number of sites & $\begin{array}{l}\text { Number of variable } \\
\text { sites }\end{array}$ & $\begin{array}{l}\text { Number of parsimony } \\
\text { information sites }\end{array}$ & Nucleotide diversity \\
\hline Complete cp genome & 152,707 & 728 & 342 & 0.00172 \\
LSC & 82,378 & 514 & 243 & 0.00223 \\
SSC & 17,582 & 162 & 74 & 0.00332 \\
IR & 26,372 & 27 & 13 & 0.00038 \\
Protein coding genes & 68,709 & 237 & 112 & 0.00129 \\
\hline
\end{tabular}

3 